Article

\title{
Geochronological, Geochemical and Sr-Nd-Hf Isotopic Studies of the A-type Granites and Adakitic Granodiorites in Western Junggar: Petrogenesis and Tectonic Implications
}

\author{
Jia Lu ${ }^{1,+}$, Chen Zhang ${ }^{2,3,+}$ and Dongdong Liu $2,4, *$ (D) \\ 1 Faculty of Land and Resource Engineering, Kunming University of Science and Technology, \\ Kunming 650093, China; chnlujia@163.com \\ 2 State Key Laboratory of Petroleum Resources and Prospecting, China University of Petroleum, \\ Beijing 102249, China; zhangchencup@163.com \\ 3 College of Geoscience, China University of Petroleum, Beijing 102249, China \\ 4 Unconventional Petroleum Research Institute, China University of Petroleum, Beijing 102249, China \\ * Correspondence: liudd@cup.edu.cn \\ + Both authors contributed equally to this work.
}

Received: 26 March 2020; Accepted: 26 April 2020; Published: 29 April 2020

\begin{abstract}
Late Carboniferous magmatism in the Western Junggar region of the Central Asian Orogenic Belt (CAOB) provides a critical geological record of regional tectonic and geodynamic history. In this study, we determined the zircon U-Pb isotopic compositions, bulk-rock Sr-Nd-Hf isotopic compositions, and major and trace element geochemistry of two granitic bodies in the Western Junggar, with the aim of constraining their emplacement ages, magmatic origin, and geodynamic significance. Radiometric ages indicate that the plutons were emplaced during the Late Carboniferous (322-307 Ma). Plutons in the North Karamay region are characterized by high Sr content (347-362 ppm) and low Y content (15.3-16.7 ppm), yielding relatively high $\mathrm{Sr} / \mathrm{Y}$ ratios (20.8-23.7). They show consistent $\mathrm{Yb}$ (1.68-1.85 ppm), Cr (16-19 ppm), Co (7.5-8.1 ppm) and Ni (5.9-6.6 ppm) content, similar to that of modern adakites. The Hongshan plutons are characterized by high $\mathrm{SiO}_{2}(69.95-74.66 \mathrm{wt} \%), \mathrm{Na}_{2} \mathrm{O}(3.26-3.64 \mathrm{wt} \%)$, and $\mathrm{K}_{2} \mathrm{O}(4.84-5.16 \mathrm{wt} \%)$ content, low $\mathrm{Al}_{2} \mathrm{O}_{3}$ (12.02-12.84 $\mathrm{wt}^{\mathrm{t}} \%$;) and $\mathrm{MgO}(0.13-018 \mathrm{wt} \%)$ content, and low $\mathrm{Mg}^{\#}$ values (0.16-0.22). This group shows a clear geochemical affinity with A-type granites. All of the studied granitoids have positive $\varepsilon \mathrm{Nd}(\mathrm{t})(+4.89$ to +7.21$)$ and $\varepsilon \mathrm{Hf}(\mathrm{t})(+7.70$ to +13.00$)$ values, with young $\left.\mathrm{T}_{\mathrm{DM}}(\mathrm{Nd}) 806-526 \mathrm{Ma}\right)$ and $\mathrm{T}_{\mathrm{DM}}(\mathrm{Hf})$ (656-383 Ma) ages, indicating a substantial addition of juvenile material. The adakitic granodiorites in the North Karamay region were likely generated via partial melting of thickened lower crust, while the A-type granites in the Hongshan area may have been derived from the melting of lower-middle crust in an intra-oceanic arc, which consists mainly of oceanic crust. The emplacement of these granitoids represents a regional magmatic "flare up", which can be explained by the rollback of a subducting slab.
\end{abstract}

Keywords: Central Asian Orogenic Belt; Western Junggar; A-type granite; adakite; subduction; slab roll-back

\section{Introduction}

Magmatic rocks represent crucial windows into regional tectonic processes and events, and can provide important constrains on the dynamics of the deep asthenosphere [1-5]. The production of subduction-related magmatic rocks can be attributed to several distinct geodynamic mechanisms, 
such as slab roll-back [6,7], slab tearing [8,9], slab break-off [10], or ridge subduction [11,12]. These processes are all related to the upwelling of hot asthenospheric mantle, which provides the heat source for magmatism [13,14] and progressively changes the composition of the magma sources [15-19].

The Central Asian Orogenic Belt (CAOB), sometimes described as the Altaid Collage or the Altaids, is situated along the margin of the Siberian, East European, Tarim, and North China cratons (Figure 1a; [5,20-29]). The CAOB formed mainly via subduction, terrane accretion, craton collision, and post-collisional extension, from the Neoproterozoic through the end of the Paleozoic [24,30-33]. Voluminous Paleozoic and Mesozoic granitoids have intruded into the CAOB over its long evolutionary history $[13,30,31,34-40]$. These granitoids have characteristically positive $\varepsilon \mathrm{Hf}(\mathrm{t})$ and $\varepsilon \mathrm{Nd}(\mathrm{t})$ values and young $\mathrm{T}_{\mathrm{DM}-\mathrm{Nd}}$ model ages $[13,20,30,41,42]$. Together, they constitute a significant proportion of the continental crust, and record the history of crustal growth and associated processes. Therefore, their ages, compositions, and petrogenesis are important for understanding the orogenic history of the CAOB.

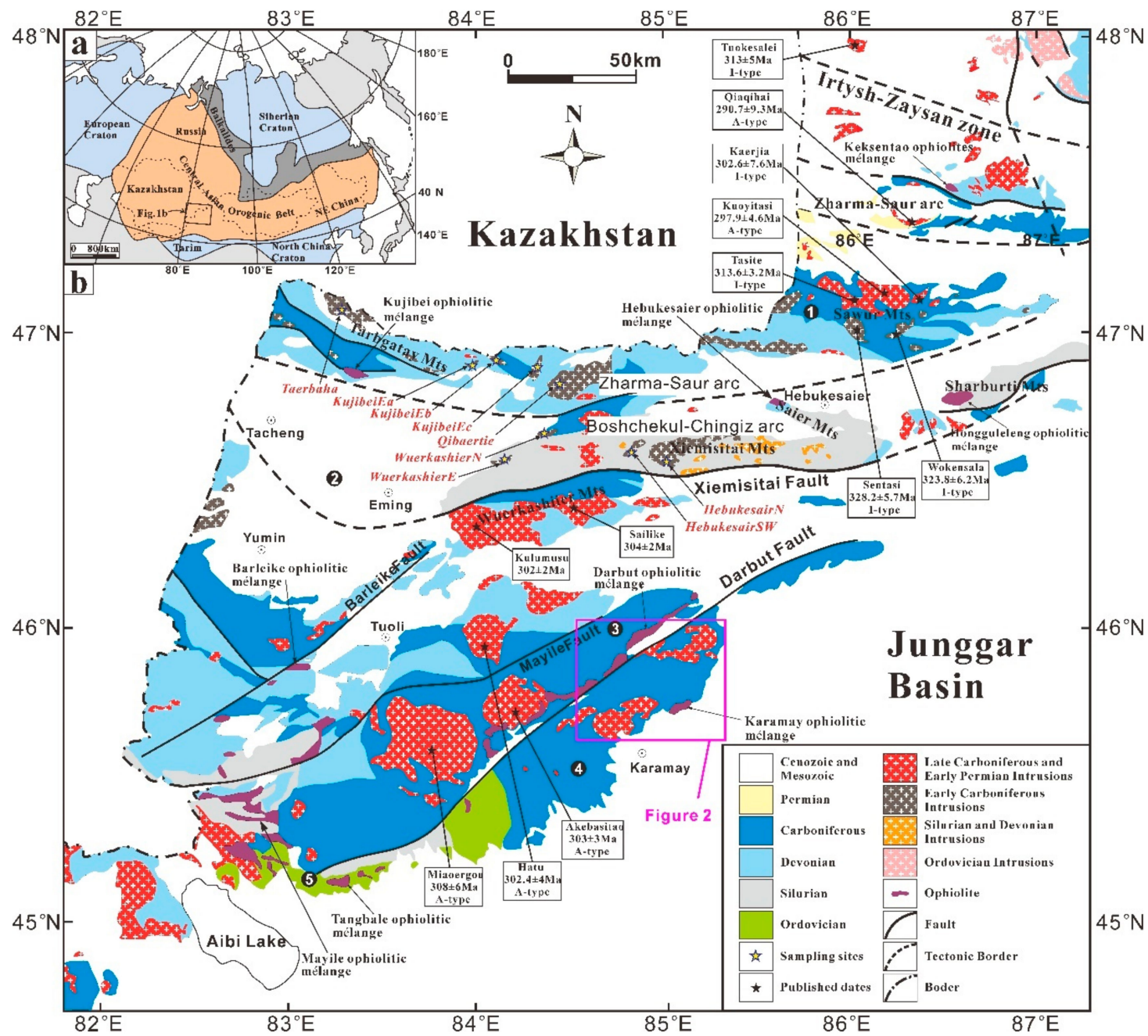

Figure 1. (a) Simplified tectonic divisions of the Central Asian Orogenic Belt (modified after Jahn et al. [5]; Yakubchuk [22]); (b) simplified geological map of the West Junggar.

The Western Junggar is located in the southern part of the CAOB, and provides an ideal natural laboratory to study the evolution of the orogen (Figure $1 b ;[23,31,43]$ ). Granitoid intrusions are widespread in the Western Junggar region, and are characterized by highly-depleted Nd isotopic signatures $(\varepsilon \mathrm{Nd}(t)=+6.4$ to +9.2$)$ [4,27-30,44,45]. However, the origin of the granitic intrusions in the Western Junggar is controversial. They have been proposed to reflect either a subduction-related island arc setting $[13,23,46-48]$, or a post-collision extensional regime $[4,44,45]$. These two models 
have significantly different implications for interpreting petrogenesis and crustal growth in the Western Junggar.

In this study, we determine the zircon U-Pb ages and $\mathrm{Sr}-\mathrm{Nd}-\mathrm{Hf}$ isotopic compositions, as well as the major and trace element geochemistry, of two granitoid plutons in the Western Junggar. The results obtained constrain the petrogenetic history of these granitoids, allowing for a deeper understanding of tectonic evolution in the southern part of the Western Junggar.

\section{Geologic Background}

The Western Junggar terrane, located in the southern part of the CAOB, is bounded to the north by the Irtysh-Zaysan accretionary complex, and to the south by the North Tian Shan accretionary complex $[13,46,49,50]$. The terrane can be divided into northern and southern sections by the Xiemisitai Fault (Figure 1; [13,49,51]).

The northern part includes the NW-trending Zharma-Saur and Boshchekul-Chingiz volcanic arcs, which host a Paleozoic sedimentary succession ranging from Cambrian to Permian in age $[49,52,53]$. The Boshchekul-Chingiz arc is characterized by a suite of Late Silurian to Early Devonian intrusions in the Xiemisitai-Saier mountain range. In contrast, intrusive rocks in the Zharma-Saur arc, such as those found in the Tarbgatay-Sauer mountains, are generally Early Carboniferous in age [49]. The two arcs are separated by the approximately East-West striking Kujibai-Hebukesaier-Hongguleleng ophiolitic 'mélange' belt, which extends westward into the West Tarbgatay ophiolite complex in eastern Kazakhstan [54]. This belt may have formed prior to the Early Carboniferous, as indicated by the presence of ophiolitic fragments in the Lower Carboniferous conglomerate overlying the Kujibai ophiolitic mélange [54].

The southern part of the Western Junggar is characterized by the NE-trending Karamay volcanic arc [55]. Carboniferous volcanic and sedimentary strata are widespread throughout the southern part of the Western Junggar, and are particularly abundant around the Darbut Fault; this volcano-sedimentary succession includes the Xibeikulasi, Baogutu, and Tailegula formations [42,51]. The Xibeikulasi Formation includes bedded mudstones, volcaniclastic siltstones, and greywackes with graded bedding. The Baogutu Formation consists of lithic-vitric felsic tuffs, volcaniclastic sandstones, and siltstones. The Tailegula Formation also contains felsic tuff, as well as pillow lavas and basalt flows with intercalated cherts. The zircon U-Pb ages of felsic tuffs in the Tailegula and Baogutu formations range from $328 \mathrm{Ma}$ [56] to $357.5 \mathrm{Ma}$ [57], and from $328 \mathrm{Ma}$ to $342 \mathrm{Ma}$ [58], respectively.

Five ophiolitic or ultramafic-to-mafic 'mélange' belts occur in the Early to Middle Paleozoic accretionary complexes in the southern part of the Western Junggar. These include the Tangbale ophiolitic mélange (531 $\pm 15 \mathrm{Ma}$ [59]), the Mayile ophiolitic mélange (415 Ma [60]), the Darbut ophiolitic 'mélange' (391 \pm 7 Ma [61]), the Karamay ophiolitic 'mélange' (307 Ma, [62]), and the Barleik ophiolitic 'mélange' [63].

The two studied plutonic suites (i.e., the Hongshan plutons and the North Karamay plutons) are located in the central part of the Western Junggar region, and close to a branch of the NE-trending Darbut Fault. They intruded into a succession of Lower Carboniferous volcanic and sedimentary rocks, which also includes basalts and some mafic and felsic dikes (Figure 2).

The Hongshan plutons are located about $40 \mathrm{~km}$ northeast of the city of Karamay. They have an elongated ovoid shape, and are approximately $12 \mathrm{~km}$ long, exposed over an area of $\sim 22 \mathrm{~km}^{2}$. Satellite imagery shows that the Hongshan plutons form a dark-colored, ring-shaped feature in the Western Junggar that is unique in the $\mathrm{CAOB}$ [64]. Various studies have yielded a range of formation ages for the Hongshan plutons, including 244.6 Ma [65], $297 \pm 12 \mathrm{Ma}$ [30], $305 \pm 4$ Ma [66], and $301 \pm 4$ Ma [67].

The North Karamy plutons are situated $20 \mathrm{~km}$ northwest of the city of Karamay, and cover an area of $310 \mathrm{~km}^{2}$. They were emplaced mainly in the interval from 321-296 Ma [40,68,69]. The massive mafic inclusions in the North Karamy plutons indicate that they experienced significant mixing with magmas of different compositions during petrogenesis $[13,70,71]$. 


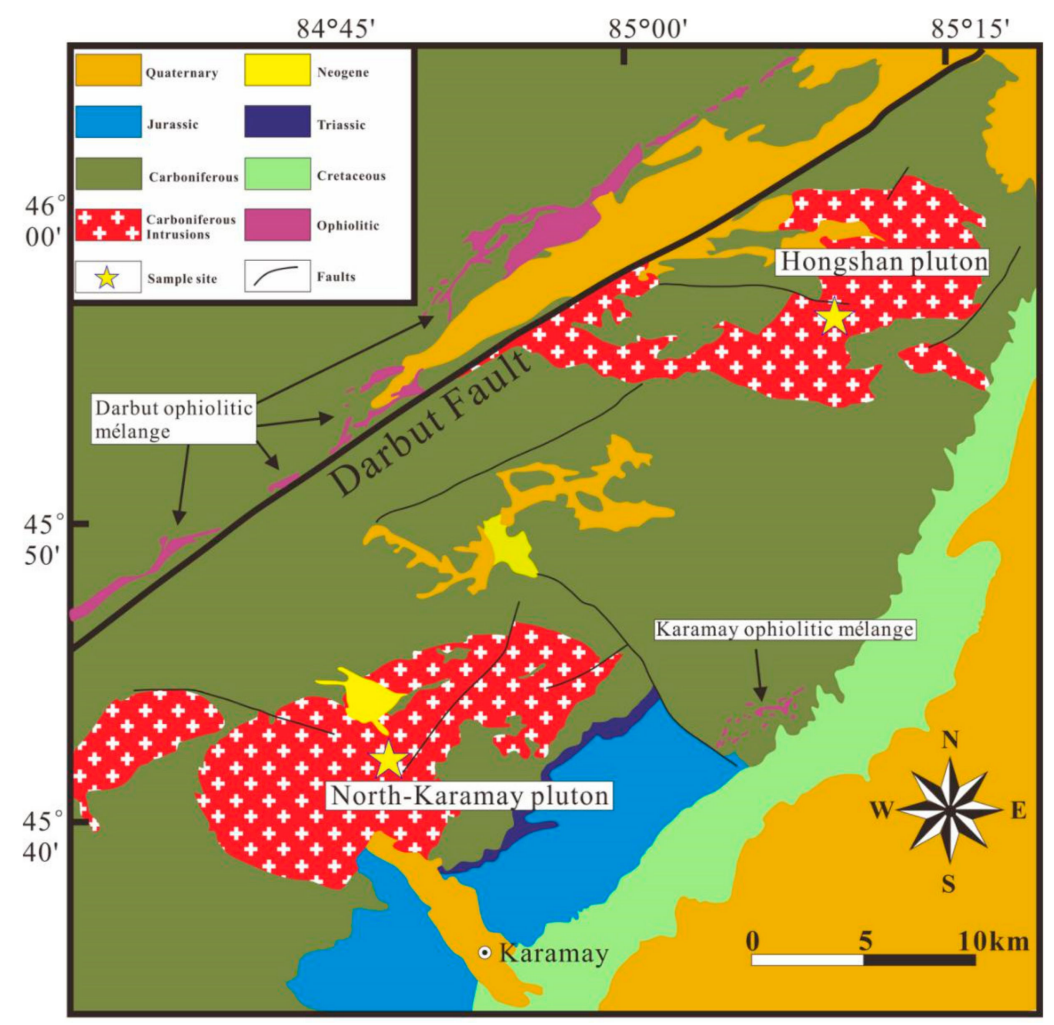

Figure 2. Simplified geological map of studied region.

\section{Sampling and Analytical Methods}

Eight samples from the granitic suites in the Karamay region were collected for this study; four samples from the North Karamay granitoids (NK-01 to NK-04), and four samples from Hongshan granitoids (HS-01 to HS-04). Specific sampling locations are shown in Figure 2; hand specimen photographs and photomicrographs of the samples are shown in Figure 3.
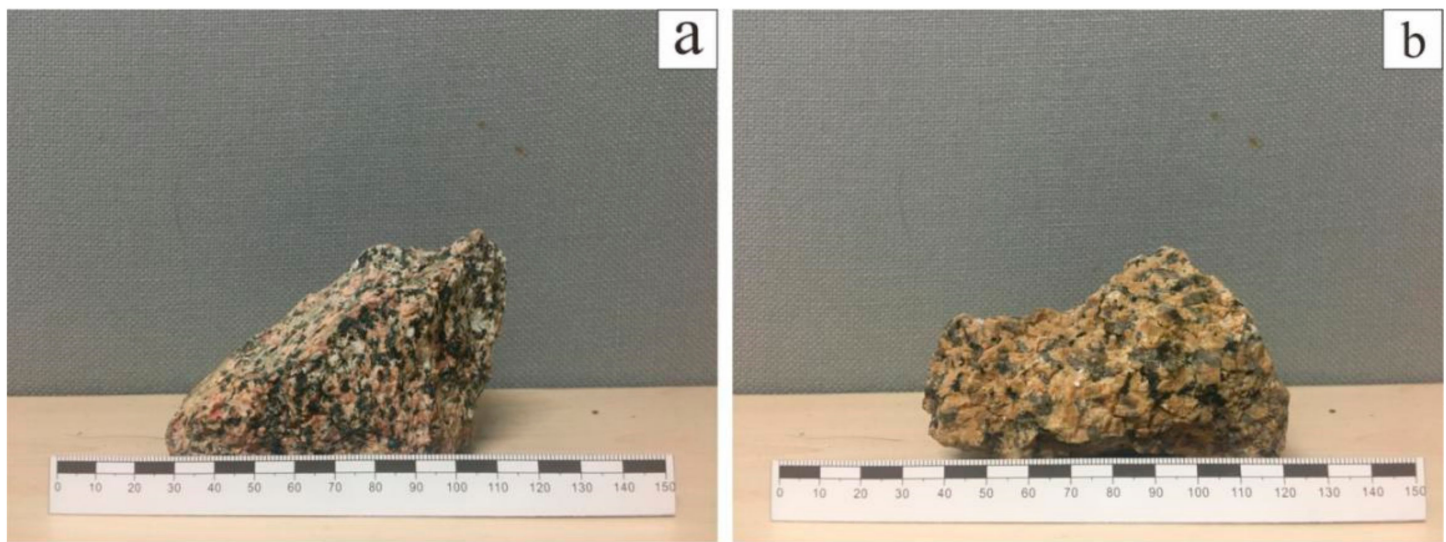

Figure 3. Photographs of the studied granitoids in the hand specimen: (a) the Hongshan pluton, (b) the North-Karamay pluton.

The North Karamay granitoids consist of plagioclase (30-35 vol.\%), K-feldspar (25-30 vol.\%), quartz (25-30 vol.\%), biotite (4-5 vol.\%), and muscovite (1 vol.\%). The Hongshan granitoids also consist predominantly of plagioclase (35-40 vol.\%), K-feldspar (20-25 vol.\%), quartz (30-35 vol.\%), biotite (4-5 vol.\%), and muscovite (1-2 vol.\%), with no significant accessory minerals. 


\subsection{Zircon $\mathrm{U}-\mathrm{Pb}$ Dating}

Zircon grains were extracted using standard density and magnetic separation techniques. Cathodoluminescence (CL) images of the separated zircon grains were obtained using an FEI NOVA NanoSEM 450 scanning electron microscope, equipped with a Gatan Mono CL4 cathodoluminescence system, at the State Key Laboratory of Continental Tectonics and Dynamics, Institute of Geology, Chinese Academy of Geological Sciences. Zircon U-Pb isotope and trace element analyses were carried out simultaneously using an Agilent 7500a ICP-MS equipped with a $193 \mathrm{~nm}$ GeoLas 2005 laser ablation system, at the State Key Laboratory of Geological Processes and Mineral Resources, China University of Geoscience, Beijing, Wuhan. Analyses were conducted with a beam diameter of $32 \mu \mathrm{m}, 5 \mathrm{~Hz}$ repetition rate, and energy of 10-20 J/ $\mathrm{cm}^{2}$. Zircon standard 91500 was analyzed twice for each five sample analyses, and used to calibrate isotope fractionation. The NIST 610 glass standard was also analyzed once for every ten analyses, in order to correct for time-dependent drift in the sensitivity or mass discrimination of the instrument. Details of the instrumental conditions, analytical procedures, and data reduction process are given in Liu et al. [72]. Calculated ages and concordia diagrams were generated using Isoplot/Ex 2.49 software [73].

\subsection{Sr-Nd Isotope Analysis}

Sample powders were spiked with mixed isotope tracers and dissolved in Teflon capsules with a mixture of $\mathrm{HF}$ and $\mathrm{HNO}_{3}$ prior to $\mathrm{Sr}$ and $\mathrm{Nd}$ isotope analysis. Sr and rare earth elements (REEs) were separated using Eichrom resin columns, with $0.1 \% \mathrm{HNO}_{3}$ as elutant. Separation of $\mathrm{Nd}$ from the REE fraction was carried out using a HDEHP column, with a $0.18 \mathrm{~N} \mathrm{HCl}$ elutant. Isotopic measurements were conducted via thermal ionization mass spectrometry (TIMS) at the Experimental Test Center, Tianjin Institute of Geology and Mineral Resources. Mass fractionation corrections for $\mathrm{Sr}$ and $\mathrm{Nd}$ isotopic ratios were based on ${ }^{86} \mathrm{Sr} /{ }^{88} \mathrm{Sr}$ and ${ }^{146} \mathrm{Nd} /{ }^{144} \mathrm{Nd}$ ratios of 0.1194 and 0.7219 , respectively. The ${ }^{87} \mathrm{Sr} /{ }^{86} \mathrm{Sr}$ ratios of the NBS987 and NBS607 isotopic standards, and the ${ }^{143} \mathrm{Nd} /{ }^{144} \mathrm{Nd}$ ratios for the BCR-1 and La Jolla isotopic standards, were measured as $0.710240 \pm 15(2 \sigma), 1.20032 \pm 30(2 \sigma)$, $0.512663 \pm 9(2 \sigma)$, and $0.511862 \pm 7(2 \sigma)$, respectively. Sample preparation and analytical procedures followed those of Zhang et al. [74].

\subsection{Major and Trace Element Analyses}

The whole rock major and trace element compositions of the studied samples were analyzed at the State Key Laboratory of Ore Deposit Geochemistry, Institute of Geochemistry, Chinese Academy of Sciences. Fresh chips of bulk sample were powdered to 200-mesh using a tungsten carbide ball mill. Subsamples for trace element analysis were digested in high-pressure Teflon bombs at $190{ }^{\circ} \mathrm{C}$ for $48 \mathrm{~h}$, using a mixture of $\mathrm{HF}$ and $\mathrm{HNO}_{3}$. Major elements were analyzed using a Rikagu RIX 2100 x-ray fluorescence (XRF) spectrometer, and trace elements were analyzed using an Agilent 7500a inductively coupled plasma mass spectrometer (ICP-MS). United States Geological Survey and international rock standards (BHVO-2, AGV-2, BCR-2 and GSP-1) were used to assess data quality and measurement repeatability. The analytical precision and accuracy for most major and trace elements were better than $5 \%$ and $10 \%$, respectively [75].

\subsection{In Situ Zircon Hf Isotope Analysis}

Zircon Hf isotope analyses were conducted using a Neptune Plus multicollector ICP-MS instrument (Thermo Fisher Scientific, Germany) coupled to a Geolas 2005 excimer ArF laser ablation system (LambdaPhysik, Göttingen, Germany), at the State Key Laboratory of Geological Processes and Mineral Resources, China University of Geosciences, Wuhan, China. All data were acquired in single-spot ablation mode, with a spot size of $44 \mu \mathrm{m}$. Each measurement consisted of $20 \mathrm{~s}$ of background signal acquisition, followed by $50 \mathrm{~s}$ of ablation signal acquisition. Detailed analytical procedures and operating conditions for both the laser ablation system and the MC-ICP-MS instrument are described 
in Hu et al. [76]. Offline selection and integration of analyte signals, and mass bias calibrations, were conducted using the ICP-MS DataCal software [77].

\section{Results}

\subsection{Zircon U-Pb Ages}

Two samples from the North Karamay and Hongshan plutons were selected for zircon U-Pb dating (Table 1). The zircon grains in these granite samples are pale yellow in color, transparent, euhedral to subhedral, and $80-100 \mu \mathrm{m}$ in size. All zircons exhibit bright cathodoluminescence, with clear concentric oscillatory zoning (Figure 4). The Th and U concentrations for all analyzed sites ranged from 129 to 2231 ppm and from 218 to 1772 ppm, respectively, with relatively high Th/U ratios (0.64-1.53). These characteristics are typical of zircons that are magmatic in origin. All U-Pb measurements plotted very close to the concordant line on the ${ }^{206} \mathrm{~Pb} /{ }^{238} \mathrm{U}$ vs. ${ }^{207} \mathrm{~Pb} /{ }^{235} \mathrm{U}$ concordia diagram (Figure 5). These analyses yield ages of $308 \pm 5$ Ma for the Hongshan granitoids, and $323 \pm 3$ Ma for the North Karamay granitoids.

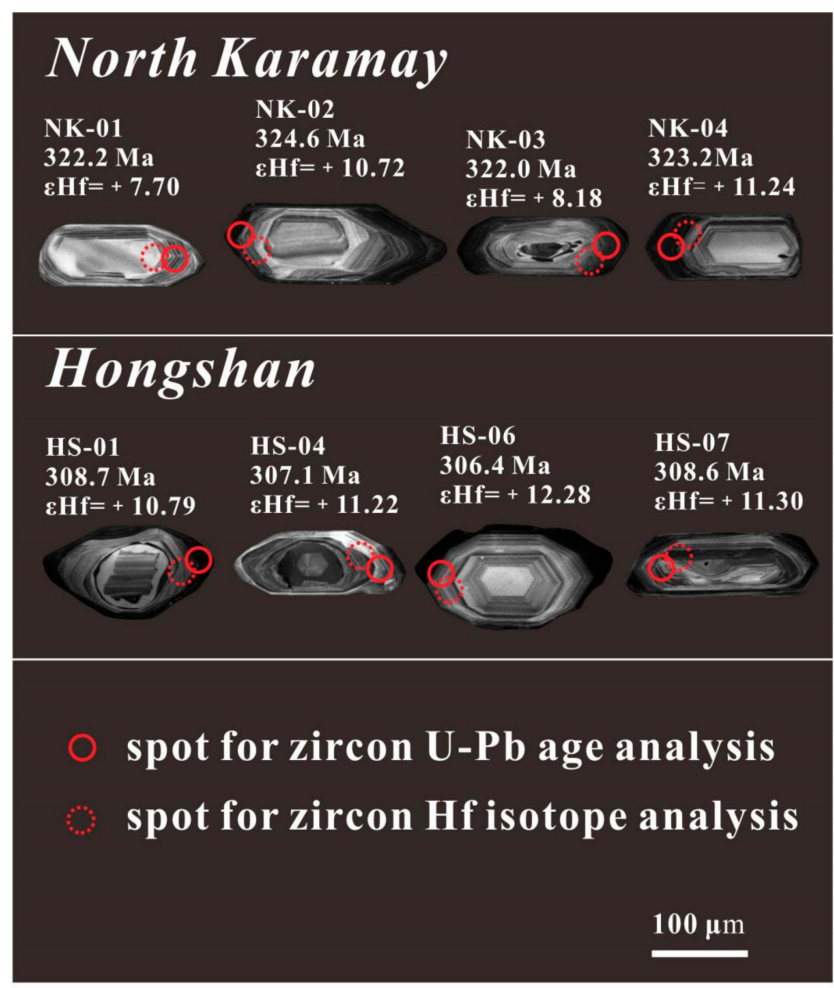

Figure 4. Representative cathodoluminescence (CL) images of zircons for the granitoids in Western Junggar. 
Table 1. U-Pb dating results of North-Karamay granitoids and Hongshan granitoids.

\begin{tabular}{|c|c|c|c|c|c|c|c|c|c|c|c|c|c|}
\hline \multirow{2}{*}{ Sample } & \multirow{2}{*}{$\mathrm{Th} / \mathrm{U}$} & \multicolumn{6}{|c|}{ Ratio } & \multicolumn{6}{|c|}{ Age (Ma) } \\
\hline & & ${ }^{207} \mathrm{~Pb} /{ }^{206} \mathrm{~Pb}$ & $1 \sigma$ & ${ }^{207} \mathrm{~Pb} /{ }^{235} \mathrm{U}$ & $1 \sigma$ & ${ }^{206} \mathrm{~Pb} /{ }^{238} \mathrm{U}$ & $1 \sigma$ & ${ }^{207} \mathrm{~Pb} /{ }^{206} \mathrm{~Pb}$ & $1 \sigma$ & ${ }^{207} \mathrm{~Pb} /{ }^{235} \mathrm{U}$ & $1 \sigma$ & ${ }^{207} \mathrm{~Pb} /{ }^{238} \mathrm{U}$ & 10 \\
\hline NK-01 & 0.48 & 0.05793 & 0.00401 & 0.37660 & 0.01522 & 0.05133 & 0.00135 & 332 & 14 & 328 & 12 & 322 & 9 \\
\hline NK-02 & 0.56 & 0.05543 & 0.00516 & 0.39083 & 0.01422 & 0.05172 & 0.00140 & 335 & 15 & 339 & 10 & 325 & 8 \\
\hline NK-03 & 0.56 & 0.05674 & 0.00308 & 0.35436 & 0.01554 & 0.05122 & 0.00167 & 311 & 14 & 309 & 11 & 322 & 10 \\
\hline NK-04 & 0.45 & 0.05371 & 0.00436 & 0.39214 & 0.01183 & 0.05141 & 0.00221 & 307 & 17 & 341 & 9 & 323 & 11 \\
\hline NK-05 & 1.22 & 0.05620 & 0.00365 & 0.37741 & 0.01228 & 0.05113 & 0.00134 & 338 & 13 & 328 & 9 & 321 & 8 \\
\hline NK-06 & 0.45 & 0.05962 & 0.00347 & 0.36680 & 0.01649 & 0.05129 & 0.00100 & 323 & 11 & 319 & 12 & 322 & 8 \\
\hline NK-07 & 0.56 & 0.05642 & 0.00395 & 0.35945 & 0.01505 & 0.05092 & 0.00150 & 317 & 18 & 313 & 11 & 320 & 12 \\
\hline NK-08 & 0.48 & 0.04817 & 0.00340 & 0.36678 & 0.01302 & 0.05122 & 0.00120 & 309 & 13 & 318 & 10 & 322 & 8 \\
\hline NK-09 & 0.56 & 0.06071 & 0.00477 & 0.37263 & 0.01295 & 0.05109 & 0.00117 & 340 & 18 & 324 & 9 & 321 & 7 \\
\hline NK-10 & 0.58 & 0.05304 & 0.00269 & 0.35749 & 0.01267 & 0.05143 & 0.00135 & 312 & 11 & 311 & 11 & 324 & 9 \\
\hline NK-11 & 0.48 & 0.05601 & 0.00367 & 0.37690 & 0.00996 & 0.05144 & 0.00135 & 335 & 13 & 328 & 8 & 324 & 9 \\
\hline NK-12 & 1.29 & 0.05778 & 0.00302 & 0.38116 & 0.01203 & 0.05136 & 0.00134 & 338 & 17 & 331 & 11 & 322 & 8 \\
\hline NK-13 & 0.44 & 0.05226 & 0.00315 & 0.36803 & 0.01681 & 0.05157 & 0.00123 & 310 & 12 & 320 & 9 & 323 & 7 \\
\hline NK-14 & 0.56 & 0.05246 & 0.00331 & 0.35096 & 0.01615 & 0.05147 & 0.00184 & 306 & 14 & 305 & 12 & 323 & 11 \\
\hline NK-15 & 0.48 & 0.05373 & 0.00290 & 0.36580 & 0.01373 & 0.05151 & 0.00140 & 309 & 13 & 318 & 10 & 323 & 8 \\
\hline NK-16 & 0.56 & 0.06201 & 0.00450 & 0.38188 & 0.01246 & 0.05189 & 0.00117 & 339 & 15 & 330 & 10 & 325 & 7 \\
\hline NK-17 & 0.58 & 0.05276 & 0.00250 & 0.37210 & 0.01164 & 0.05139 & 0.00140 & 306 & 11 & 324 & 10 & 323 & 8 \\
\hline NK-18 & 0.5 & 0.05826 & 0.00431 & 0.37573 & 0.01337 & 0.05180 & 0.00144 & 335 & 15 & 327 & 13 & 325 & 9 \\
\hline NK-19 & 1.41 & 0.05624 & 0.00260 & 0.38288 & 0.01793 & 0.05165 & 0.00090 & 336 & 13 & 332 & 13 & 324 & 6 \\
\hline NK-20 & 0.45 & 0.05083 & 0.00281 & 0.37144 & 0.01153 & 0.05143 & 0.00100 & 322 & 12 & 323 & 8 & 323 & 6 \\
\hline NK-21 & 0.56 & 0.05195 & 0.00274 & 0.36601 & 0.01419 & 0.05160 & 0.00108 & 311 & 13 & 318 & 11 & 324 & 9 \\
\hline NK-22 & 0.47 & 0.05267 & 0.00373 & 0.36695 & 0.00978 & 0.05109 & 0.00210 & 309 & 15 & 320 & 10 & 320 & 9 \\
\hline NK-23 & 0.57 & 0.05723 & 0.00517 & 0.38022 & 0.01231 & 0.05146 & 0.00105 & 337 & 16 & 329 & 9 & 323 & 7 \\
\hline NK-24 & 0.57 & 0.05191 & 0.00287 & 0.36345 & 0.01710 & 0.05124 & 0.00100 & 313 & 13 & 315 & 12 & 322 & 10 \\
\hline NK-25 & 0.47 & 0.05555 & 0.00402 & 0.37931 & 0.01400 & 0.05125 & 0.00200 & 334 & 14 & 329 & 11 & 322 & 10 \\
\hline NK-26 & 1.24 & 0.05926 & 0.00328 & 0.37074 & 0.01329 & 0.05065 & 0.00053 & 335 & 15 & 323 & 10 & 318 & 7 \\
\hline NK-27 & 0.44 & 0.05371 & 0.00316 & 0.36528 & 0.02167 & 0.05152 & 0.00160 & 316 & 13 & 317 & 10 & 324 & 8 \\
\hline NK-28 & 0.57 & 0.05410 & 0.00345 & 0.36126 & 0.01803 & 0.05089 & 0.00120 & 310 & 14 & 314 & 11 & 320 & 12 \\
\hline
\end{tabular}


Table 1. Cont.

\begin{tabular}{|c|c|c|c|c|c|c|c|c|c|c|c|c|c|}
\hline \multirow{2}{*}{ Sample } & \multirow{2}{*}{$\mathrm{Th} / \mathrm{U}$} & \multicolumn{6}{|c|}{ Ratio } & \multicolumn{6}{|c|}{ Age (Ma) } \\
\hline & & ${ }^{207} \mathrm{~Pb} /{ }^{206} \mathrm{~Pb}$ & $1 \sigma$ & ${ }^{207} \mathrm{~Pb} /{ }^{235} \mathrm{U}$ & $1 \sigma$ & ${ }^{206} \mathrm{~Pb} /{ }^{238} \mathrm{U}$ & $1 \sigma$ & ${ }^{207} \mathrm{~Pb} /{ }^{206} \mathrm{~Pb}$ & $1 \sigma$ & ${ }^{207} \mathrm{~Pb} /{ }^{235} \mathrm{U}$ & $1 \sigma$ & ${ }^{207} \mathrm{~Pb} /{ }^{238} \mathrm{U}$ & 10 \\
\hline NK-29 & 0.48 & 0.05510 & 0.00351 & 0.36536 & 0.01144 & 0.05141 & 0.00150 & 333 & 15 & 318 & 10 & 323 & 9 \\
\hline NK-30 & 0.56 & 0.06185 & 0.00490 & 0.37612 & 0.00996 & 0.05116 & 0.00105 & 339 & 17 & 327 & 9 & 322 & 7 \\
\hline NK-31 & 0.58 & 0.05441 & 0.00273 & 0.36198 & 0.01244 & 0.05140 & 0.00120 & 311 & 14 & 315 & 10 & 323 & 9 \\
\hline NK-32 & 0.48 & 0.05554 & 0.02513 & 0.38276 & 0.02745 & 0.05134 & 0.00167 & 334 & 13 & 329 & 12 & 323 & 10 \\
\hline NK-33 & 1.28 & 0.06239 & 0.01986 & 0.37584 & 0.03038 & 0.05139 & 0.00120 & 338 & 16 & 327 & 11 & 323 & 8 \\
\hline NK-34 & 0.44 & 0.05642 & 0.02111 & 0.37108 & 0.01908 & 0.05127 & 0.00117 & 317 & 13 & 322 & 9 & 322 & 7 \\
\hline NK-35 & 0.56 & 0.04817 & 0.02336 & 0.36220 & 0.02434 & 0.05162 & 0.00192 & 309 & 15 & 314 & 13 & 324 & 12 \\
\hline HS-01 & 0.78 & 0.05589 & 0.00408 & 0.35507 & 0.01174 & 0.04943 & 0.00243 & 313 & 12 & 310 & 12 & 310 & 10 \\
\hline HS-04 & 1.21 & 0.05287 & 0.00393 & 0.35785 & 0.01368 & 0.04901 & 0.00126 & 311 & 11 & 310 & 10 & 308 & 8 \\
\hline HS-06 & 1.13 & 0.05250 & 0.00617 & 0.35496 & 0.01282 & 0.04879 & 0.00094 & 314 & 14 & 308 & 9 & 307 & 9 \\
\hline HS-07 & 0.78 & 0.05385 & 0.00380 & 0.35815 & 0.01018 & 0.04927 & 0.00128 & 315 & 11 & 311 & 8 & 310 & 6 \\
\hline HS-08 & 0.77 & 0.05209 & 0.00663 & 0.35312 & 0.01462 & 0.04884 & 0.00078 & 309 & 13 & 308 & 11 & 307 & 10 \\
\hline HS-11 & 1.21 & 0.05349 & 0.00385 & 0.35565 & 0.01951 & 0.04876 & 0.00168 & 312 & 13 & 309 & 9 & 307 & 8 \\
\hline HS-13 & 1.1 & 0.05477 & 0.00535 & 0.35854 & 0.01803 & 0.04923 & 0.00104 & 315 & 15 & 312 & 11 & 310 & 10 \\
\hline HS-14 & 0.78 & 0.05707 & 0.00338 & 0.35461 & 0.00915 & 0.04896 & 0.00124 & 312 & 14 & 309 & 8 & 308 & 7 \\
\hline HS-15 & 0.76 & 0.05379 & 0.00757 & 0.35615 & 0.01106 & 0.04887 & 0.00141 & 312 & 11 & 310 & 10 & 308 & 9 \\
\hline HS-18 & 1.2 & 0.05461 & 0.00377 & 0.35697 & 0.01244 & 0.04895 & 0.00112 & 314 & 13 & 311 & 10 & 308 & 8 \\
\hline HS-20 & 1.07 & 0.05239 & 0.00497 & 0.35907 & 0.02516 & 0.04905 & 0.00140 & 313 & 12 & 309 & 11 & 309 & 8 \\
\hline HS-21 & 0.77 & 0.05020 & 0.00311 & 0.35589 & 0.02209 & 0.04910 & 0.00081 & 318 & 12 & 310 & 8 & 309 & 5 \\
\hline HS-22 & 0.78 & 0.05245 & 0.00362 & 0.35799 & 0.02332 & 0.04930 & 0.00157 & 314 & 13 & 311 & 11 & 310 & 9 \\
\hline HS-25 & 1.21 & 0.05403 & 0.00371 & 0.35833 & 0.01685 & 0.04901 & 0.00135 & 317 & 15 & 311 & 9 & 308 & 8 \\
\hline HS-27 & 1.11 & 0.05332 & 0.00543 & 0.35626 & 0.00764 & 0.04878 & 0.00236 & 314 & 10 & 310 & 10 & 307 & 9 \\
\hline HS-28 & 0.78 & 0.05158 & 0.00329 & 0.35395 & 0.01217 & 0.04895 & 0.00074 & 311 & 11 & 308 & 8 & 308 & 7 \\
\hline HS-29 & 0.76 & 0.05239 & 0.00632 & 0.35721 & 0.00875 & 0.04910 & 0.00157 & 315 & 13 & 311 & 10 & 308 & 9 \\
\hline HS-32 & 1.22 & 0.05607 & 0.00363 & 0.36046 & 0.00618 & 0.04894 & 0.00140 & 317 & 13 & 314 & 9 & 308 & 8 \\
\hline HS-34 & 1.09 & 0.05354 & 0.00539 & 0.35640 & 0.00824 & 0.04892 & 0.00094 & 315 & 12 & 310 & 11 & 308 & 9 \\
\hline HS-35 & 0.78 & 0.05252 & 0.00349 & 0.35598 & 0.01987 & 0.04892 & 0.00093 & 312 & 13 & 310 & 10 & 307 & 7 \\
\hline
\end{tabular}



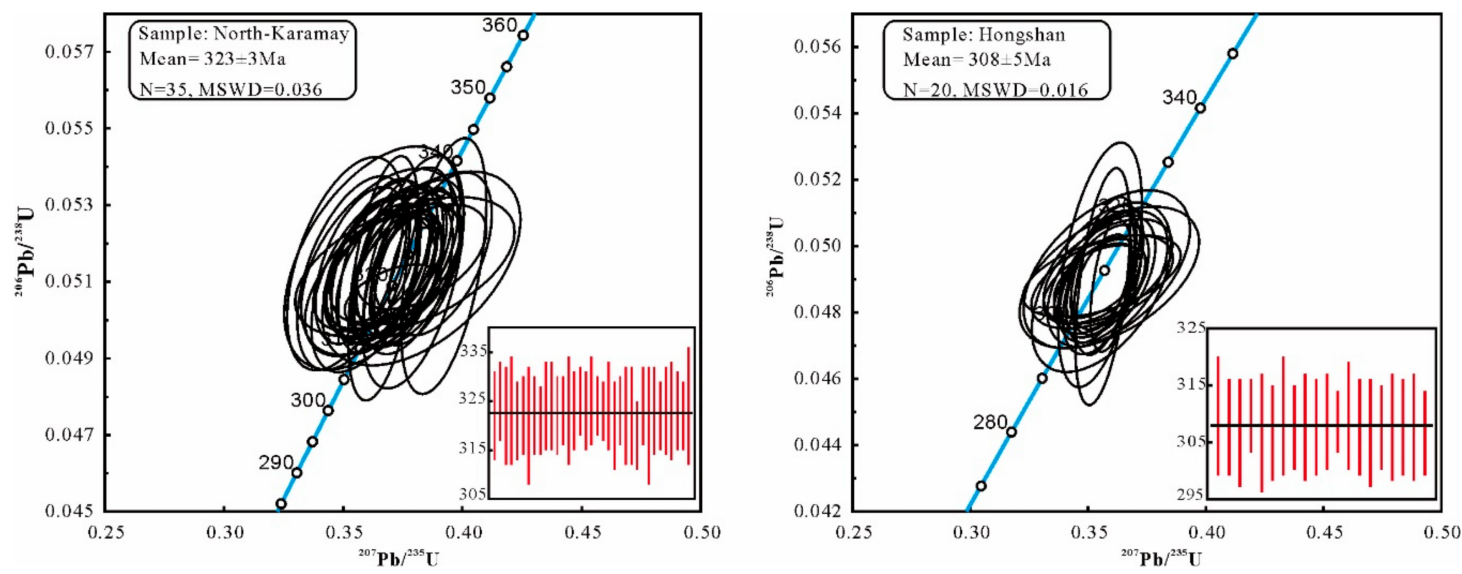

Figure 5. U-Pb concordia diagrams showing zircon ages obtained by laser-ablation inductively-coupledplasma mass spectrometry (LA-ICP-MS) for the granitic batholiths in North Junggar.

\subsection{Major and Trace Element Composition}

\subsubsection{North Karamay Granitoids}

The North Karamay granitoids are characterized by intermediate $\mathrm{SiO}_{2}$ content (Figure 6a, 65.71-66.29 wt\%; avg. $66.01 \mathrm{wt} \%)$ and calc-alkaline. They have comparatively low $\mathrm{K}_{2} \mathrm{O}$ content (2.57-2.65 wt\%; avg. 2.62 wt\%), but high $\mathrm{Al}_{2} \mathrm{O}_{3}$ (Figure 6b, 15.73-16.17 wt\%; avg. $15.88 \mathrm{wt} \%$ ), $\mathrm{Na}_{2} \mathrm{O}(4.60-4.72 \mathrm{wt} \%)$, and $\mathrm{MgO}(1.48-1.55 \mathrm{wt} \%)$ content, and high $\mathrm{Mg}^{\#}$ values (0.37-0.38). The low $\mathrm{K}_{2} \mathrm{O} / \mathrm{Na}_{2} \mathrm{O}$ ratios (generally from $0.54-0.58$ ) suggest a potassium-poor composition. These granitoids are relatively rich in $\mathrm{Co}$ (generally from 7.5-8.1 ppm), $\mathrm{Cr}$ (generally from 16-17 ppm) and Ni (generally from 5.9-6.6 ppm). They are enriched in Sr (347-362 ppm) and Ba (610-690 ppm), depleted in HREEs (e.g., $\mathrm{Yb}=1.68-1.85 \mathrm{ppm}$ ) and have high $\mathrm{Sr} / \mathrm{Y}$ ratios (20.8-23.7), which are comparable to those of modern adakites [78-80]. The North Karamay granitoids are also enriched in $\mathrm{Rb}, \mathrm{Ba}$, and $\mathrm{Pb}$, and depleted in $\mathrm{Nb}$ and $\mathrm{Zr}$ (Figure 7c,d).
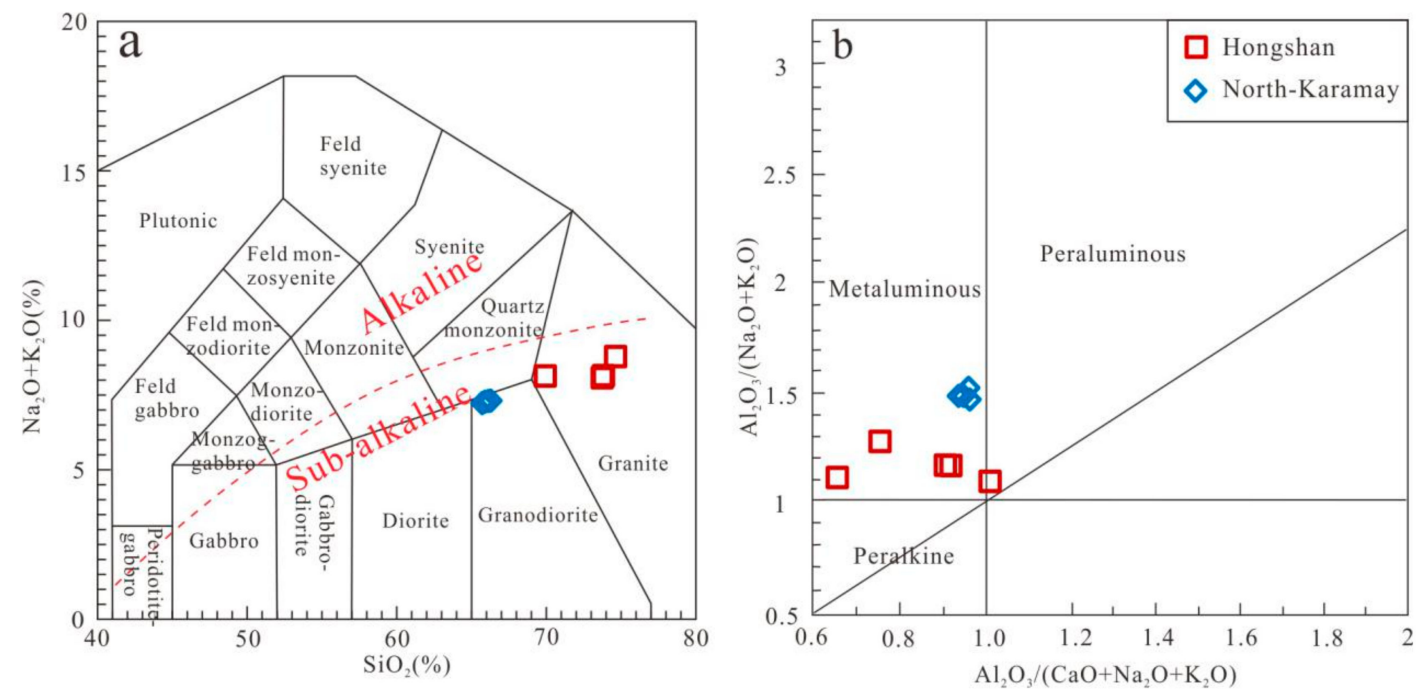

Figure 6. Major and trace element diagrams of the granitoids: (a) $\mathrm{Na}_{2} \mathrm{O}+\mathrm{K}_{2} \mathrm{O}$ vs. $\mathrm{SiO}_{2}$ diagram (after Middlemost [81]); (b) $\mathrm{Al}_{2} \mathrm{O}_{3} /\left(\mathrm{Na}_{2} \mathrm{O}+\mathrm{K}_{2} \mathrm{O}\right)$ vs. $\mathrm{Al}_{2} \mathrm{O}_{3} /\left(\mathrm{CaO}+\mathrm{Na}_{2} \mathrm{O}+\mathrm{K}_{2} \mathrm{O}\right.$ ) diagram (after Maniar and Piccoli. [82]). 

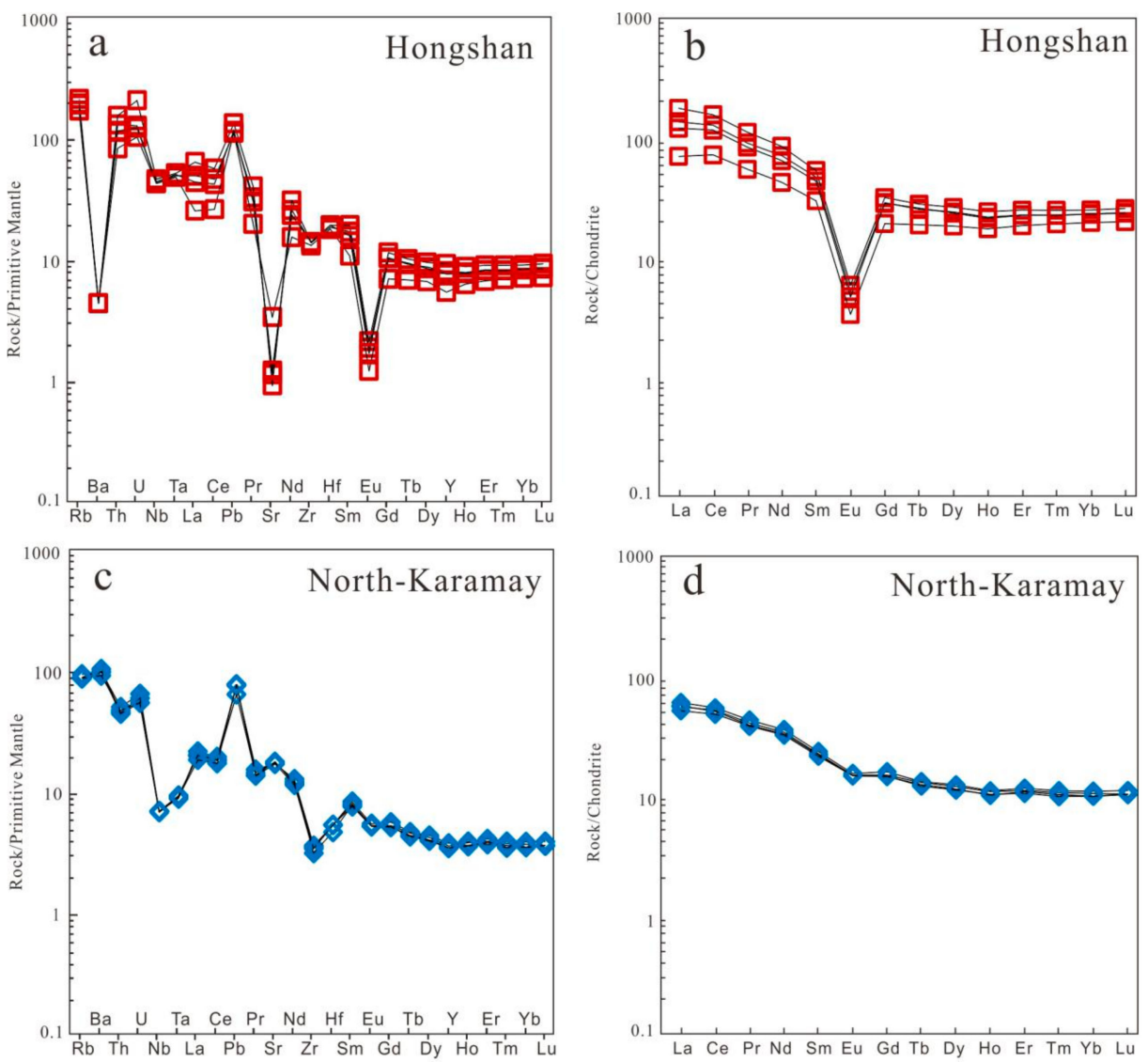

Figure 7. Primitive mantle-normalized trace element spider diagram $(\mathbf{a}, \mathbf{c})$ and chondrite-normalized REE diagram $(\mathbf{b}, \mathbf{d})$ for the studied granitoids in Western Junggar.Data of primitive mantle and chondrite are from Sun and McDonough [83].

\subsubsection{Hongshan Granitoids}

The samples from the Hongshan region are metaluminous, with $\mathrm{A} / \mathrm{CNK}$ (molar $\mathrm{Al}_{2} \mathrm{O}_{3} / \mathrm{CaO}+$ $\mathrm{Na}_{2} \mathrm{O}+\mathrm{K}_{2} \mathrm{O}$ ) ratios of $<1.1$ (Figure $6 \mathrm{~b}$ ). They have high $\mathrm{SiO}_{2}$ content $(69.95-74.66 \mathrm{wt} \%$ ), and are enriched in $\mathrm{Na}_{2} \mathrm{O}(3.26-3.64 \mathrm{wt} \%)$ and $\mathrm{K}_{2} \mathrm{O}(4.84-5.16 \mathrm{wt} \%)$, but have low $\mathrm{Al}_{2} \mathrm{O}_{3}(12.02-12.84 \mathrm{wt} \%$; avg. $12.51 \mathrm{wt} \%)$ and $\mathrm{MgO}(0.13-0.18 \mathrm{wt} \%)$ content, and low $\mathrm{Mg}^{\#}$ values $(0.16-0.22)$. The samples show similar chondrite-normalized REE distributions, characterized by relative enrichment in LREEs $\left((\mathrm{La} / \mathrm{Yb})_{\mathrm{N}}=6.1-12.2\right)$ and very low HREE content $\left((\mathrm{Tb} / \mathrm{Yb})_{\mathrm{N}}=0.23-0.27\right)$. The negative Eu anomaly seen in the Hongshan granitoids (Figure 7a,b) likely implies plagioclase differentiation during petrogenesis [30]. Primitive mantle-normalized trace element spider diagrams (Figure 7a) show that the Hongshan samples are enriched in $\mathrm{Rb}, \mathrm{U}$, and $\mathrm{Pb}$, with pronounced negative $\mathrm{Ba}, \mathrm{Sr}$, and $\mathrm{Eu}$ anomalies. The major and trace elements of the studied grantoids are list in Tables 2 and 3.

Table 2. The major chemical compositions (in $w \mathrm{t} \%$ ) and calculated parameters of the North-Karamay granitoids and the Hongshan granitoids.

\begin{tabular}{ccccccccc}
\hline Sample Number & NK-01 & NK-02 & NK-03 & NK-04 & HS-01 & HS-02 & HS-03 & HS-04 \\
\hline $\mathrm{SiO}_{2}$ & 65.90 & 66.29 & 66.13 & 65.71 & 69.95 & 73.77 & 73.87 & 74.66 \\
$\mathrm{Al}_{2} \mathrm{O}_{3}$ & 16.17 & 15.85 & 15.78 & 15.73 & 12.02 & 12.54 & 12.63 & 12.84 \\
$\mathrm{TFe}_{2} \mathrm{O}_{3}$ & 4.36 & 4.48 & 4.55 & 4.78 & 1.40 & 1.47 & 1.07 & 1.36 \\
$\mathrm{MgO}$ & 1.48 & 1.48 & 1.55 & 1.55 & 0.15 & 0.18 & 0.17 & 0.13 \\
$\mathrm{CaO}$ & 3.39 & 3.29 & 3.40 & 3.29 & 4.17 & 1.72 & 1.62 & 0.57 \\
$\mathrm{Na}_{2} \mathrm{O}$ & 4.72 & 4.73 & 4.67 & 4.60 & 3.28 & 3.26 & 3.28 & 3.64 \\
$\mathrm{~K}_{2} \mathrm{O}$ & 2.57 & 2.60 & 2.64 & 2.65 & 4.87 & 4.84 & 4.89 & 5.16 \\
$\mathrm{MnO}$ & 0.11 & 0.11 & 0.11 & 0.12 & 0.10 & 0.08 & 0.06 & 0.02 \\
\hline
\end{tabular}


Table 2. Cont

\begin{tabular}{ccccccccc}
\hline Sample Number & NK-01 & NK-02 & NK-03 & NK-04 & HS-01 & HS-02 & HS-03 & HS-04 \\
\hline $\mathrm{P}_{2} \mathrm{O}_{5}$ & 0.15 & 0.15 & 0.16 & 0.15 & 0.01 & 0.01 & 0.01 & 0.01 \\
$\mathrm{TiO}_{2}$ & 0.55 & 0.57 & 0.58 & 0.57 & 0.18 & 0.20 & 0.20 & 0.20 \\
$\mathrm{LOI}$ & 0.67 & 0.59 & 0.67 & 0.65 & 3.83 & 1.88 & 1.89 & 0.77 \\
$\mathrm{Total}$ & 100.20 & 100.26 & 100.37 & 99.92 & 99.98 & 99.95 & 99.69 & 99.36 \\
$\mathrm{~A} / \mathrm{CNK}$ & 0.97 & 0.96 & 0.94 & 0.96 & 0.66 & 0.91 & 0.93 & 1.02 \\
$\mathrm{~A} / \mathrm{NK}$ & 1.53 & 1.49 & 1.50 & 1.51 & 1.13 & 1.18 & 1.18 & 1.11 \\
$\mathrm{Mg}^{\#}$ & 0.38 & 0.37 & 0.38 & 0.37 & 0.16 & 0.18 & 0.22 & 0.15 \\
$\mathrm{~K}_{2} \mathrm{O}+\mathrm{Na}_{2} \mathrm{O}$ & 4.87 & 4.88 & 4.83 & 4.75 & 3.29 & 3.27 & 3.29 & 3.65 \\
$\mathrm{Na}_{2} \mathrm{O} / \mathrm{K}_{2} \mathrm{O}$ & 0.03 & 0.03 & 0.03 & 0.03 & 0.00 & 0.00 & 0.00 & 0.00 \\
\hline Note: $\mathrm{A}=\mathrm{Al}_{2} \mathrm{O}_{3}, \mathrm{C}=\mathrm{CaO}, \mathrm{N}=\mathrm{Na}_{2} \mathrm{O}, \mathrm{K}=\mathrm{K}_{2} \mathrm{O}$ (all in molar proportion), $\mathrm{Mg}^{\#}=100 \mathrm{Mg}^{2+} /\left(\mathrm{Mg}^{2+}+\mathrm{Fe}^{2+}\right)$.
\end{tabular}

Table 3. Trace element compositions (in ppm) and parameters of the North-Karamay granitoids and the Hongshan granitoids.

\begin{tabular}{|c|c|c|c|c|c|c|c|c|}
\hline Sample Number & NK-01 & NK-02 & NK-03 & NK-04 & HS-01 & HS-02 & HS-03 & HS-04 \\
\hline $\mathrm{Li}$ & 13.1 & 12.5 & 15.2 & 16.6 & 18.3 & 22.9 & 22.4 & 26.0 \\
\hline $\mathrm{Be}$ & 1.17 & 1.25 & 1.28 & 1.23 & 3.87 & 4.03 & 3.86 & 4.23 \\
\hline Sc & 8.4 & 9.5 & 9.6 & 9.3 & 3.2 & 3.8 & 3.9 & 4.3 \\
\hline $\mathrm{V}$ & 64 & 66 & 67 & 68 & 4 & 4 & 4 & 3 \\
\hline $\mathrm{Cr}$ & 17 & 17 & 19 & 16 & 6 & 4 & 6 & 6 \\
\hline $\mathrm{Co}$ & 7.6 & 7.5 & 7.9 & 8.1 & 0.2 & 0.2 & 0.2 & 0.3 \\
\hline $\mathrm{Ni}$ & 5.9 & 6.3 & 6.1 & 6.6 & 0.5 & 0.5 & 0.8 & 0.9 \\
\hline $\mathrm{Cu}$ & 19.7 & 18.3 & 21.7 & 22.3 & 0.9 & 1.3 & 0.8 & 0.8 \\
\hline $\mathrm{Zn}$ & 68 & 71 & 78 & 79 & 40 & 43 & 33 & 28 \\
\hline $\mathrm{Ga}$ & 18.10 & 17.95 & 18.35 & 17.90 & 15.50 & 17.05 & 16.60 & 17.75 \\
\hline $\mathrm{Ge}$ & 0.22 & 0.26 & 0.26 & 0.25 & 0.16 & 0.17 & 0.16 & 0.16 \\
\hline As & 6.8 & 7.2 & 9.9 & 10.2 & 4.2 & 4.2 & 2.4 & 4.3 \\
\hline $\mathrm{Rb}$ & 53.9 & 52.5 & 56.8 & 53.2 & 104.5 & 120.1 & 122.5 & 137.5 \\
\hline $\mathrm{Sr}$ & 362 & 347 & 348 & 357 & 69.6 & 25.3 & 23.5 & 19.1 \\
\hline Y & 15.3 & 15.3 & 16.7 & 15.8 & 24.1 & 33.9 & 35.7 & 41.1 \\
\hline $\mathrm{Zr}$ & 34.1 & 37.1 & 39.2 & 38.2 & 143.5 & 153.0 & 150.5 & 152.5 \\
\hline $\mathrm{Nb}$ & 4.8 & 4.7 & 4.8 & 4.8 & 29.6 & 30.6 & 29.7 & 32.0 \\
\hline Mo & 1.35 & 1.15 & 1.42 & 1.27 & 2.79 & 2.72 & 2.64 & 3.05 \\
\hline $\mathrm{Ag}$ & 0.06 & 0.07 & 0.08 & 0.10 & 0.03 & 0.01 & $<0.01$ & $<0.01$ \\
\hline $\mathrm{Cd}$ & 0.08 & 0.20 & 0.14 & 0.15 & 0.05 & 0.03 & 0.03 & 0.05 \\
\hline In & 0.049 & 0.065 & 0.071 & 0.072 & 0.066 & 0.073 & 0.064 & 0.064 \\
\hline $\mathrm{Sb}$ & 0.57 & 0.88 & 1.03 & 0.81 & 1.54 & 1.39 & 1.22 & 1.33 \\
\hline Cs & 1.58 & 1.99 & 1.52 & 1.59 & 1.47 & 1.74 & 1.68 & 2.05 \\
\hline $\mathrm{Ba}$ & 650 & 610 & 660 & 690 & 30 & 30 & 30 & 30 \\
\hline $\mathrm{La}$ & 13.4 & 12.2 & 14.3 & 13.2 & 17.0 & 29.2 & 33.0 & 42.7 \\
\hline $\mathrm{Ce}$ & 31.1 & 29.5 & 33.3 & 31.8 & 45.1 & 72.6 & 80.0 & 97.5 \\
\hline $\operatorname{Pr}$ & 3.68 & 3.63 & 4.08 & 3.84 & 5.31 & 8.13 & 8.80 & 10.80 \\
\hline $\mathrm{Nd}$ & 15.4 & 15.0 & 16.7 & 15.9 & 20.3 & 30.9 & 33.9 & 40.6 \\
\hline $\mathrm{Sm}$ & 3.35 & 3.27 & 3.58 & 3.43 & 4.68 & 6.81 & 7.38 & 8.36 \\
\hline $\mathrm{Eu}$ & 0.85 & 0.85 & 0.89 & 0.86 & 0.20 & 0.28 & 0.31 & 0.35 \\
\hline $\mathrm{Gd}$ & 2.98 & 2.97 & 3.25 & 3.05 & 4.05 & 5.98 & 6.05 & 6.67 \\
\hline $\mathrm{Tb}$ & 0.45 & 0.46 & 0.49 & 0.48 & 0.72 & 0.99 & 0.97 & 1.07 \\
\hline Dy & 2.83 & 2.88 & 3.13 & 3.01 & 4.78 & 6.12 & 6.26 & 6.83 \\
\hline Ho & 0.58 & 0.57 & 0.62 & 0.61 & 1.01 & 1.24 & 1.26 & 1.40 \\
\hline Er & 1.73 & 1.79 & 1.91 & 1.83 & 3.14 & 3.84 & 3.86 & 4.22 \\
\hline $\mathrm{Tm}$ & 0.25 & 0.26 & 0.28 & 0.27 & 0.50 & 0.59 & 0.59 & 0.65 \\
\hline $\mathrm{Yb}$ & 1.68 & 1.68 & 1.85 & 1.77 & 3.42 & 4.04 & 4.01 & 4.35 \\
\hline $\mathrm{Lu}$ & 0.26 & 0.26 & 0.28 & 0.26 & 0.52 & 0.61 & 0.62 & 0.67 \\
\hline Hf & 1.4 & 1.6 & 1.6 & 1.6 & 5.4 & 5.8 & 5.6 & 5.8 \\
\hline $\mathrm{Ta}$ & 0.36 & 0.36 & 0.35 & 0.37 & 1.92 & 2.01 & 1.97 & 2.06 \\
\hline W & 15.7 & 16.5 & 16.3 & 15.3 & 1.3 & 1.2 & 1.3 & 1.3 \\
\hline $\mathrm{Tl}$ & 0.19 & 0.18 & 0.20 & 0.20 & 0.55 & 0.53 & 0.51 & 0.62 \\
\hline $\mathrm{Pb}$ & 11.3 & 13.7 & 13.4 & 13.6 & 20.0 & 20.8 & 20.0 & 23.9 \\
\hline $\mathrm{Bi}$ & 0.10 & 0.11 & 0.13 & 0.13 & 0.04 & 0.03 & 0.03 & 0.02 \\
\hline Th & 3.61 & 3.85 & 4.10 & 3.71 & 6.84 & 9.54 & 10.75 & 12.70 \\
\hline $\mathrm{U}$ & 1.1 & 1.1 & 1.3 & 1.2 & 2.1 & 2.5 & 2.6 & 4.2 \\
\hline
\end{tabular}




\subsection{Sr-Nd-Hf Isotopic Composition}

The Hongshan and North Karamay granitoids have low initial ${ }^{87} \mathrm{Sr} /{ }^{86} \mathrm{Sr}$ ratios $(0.700912-0.705262)$ and ${ }^{143} \mathrm{Nd} /{ }^{144} \mathrm{Nd}$ ratios $(0.51274-0.51286)$, as well as positive $\varepsilon \mathrm{Nd}(\mathrm{t})$ values $(4.86-7.21)$. They have young $\mathrm{Nd}$ model ages, when calculated based on zircon $\mathrm{U}-\mathrm{Pb}$ ages of $307 \mathrm{Ma}$ and $322 \mathrm{Ma}$ (Figure 8a and Table 4), respectively. All zircons from the two studied granitoids have very low ${ }^{176} \mathrm{Lu} /{ }^{177} \mathrm{Hf}$ ratios $(0.00041-0.00626$, mean $=0.00383$; Table 5$)$, indicating limited radiogenic Hf production over the lifetime of these zircons. The initial ${ }^{176} \mathrm{Hf} /{ }^{177} \mathrm{Hf}$ ratios of the 30 examined zircons range from 0.28279 to 0.28298 , with $\varepsilon \mathrm{Hf}(\mathrm{t})$ values from 7.6 to13.9 (Figure 8b; Table 5). The highest $\varepsilon \mathrm{Hf}(\mathrm{t})$ values fall below the depleted mantle evolution line.
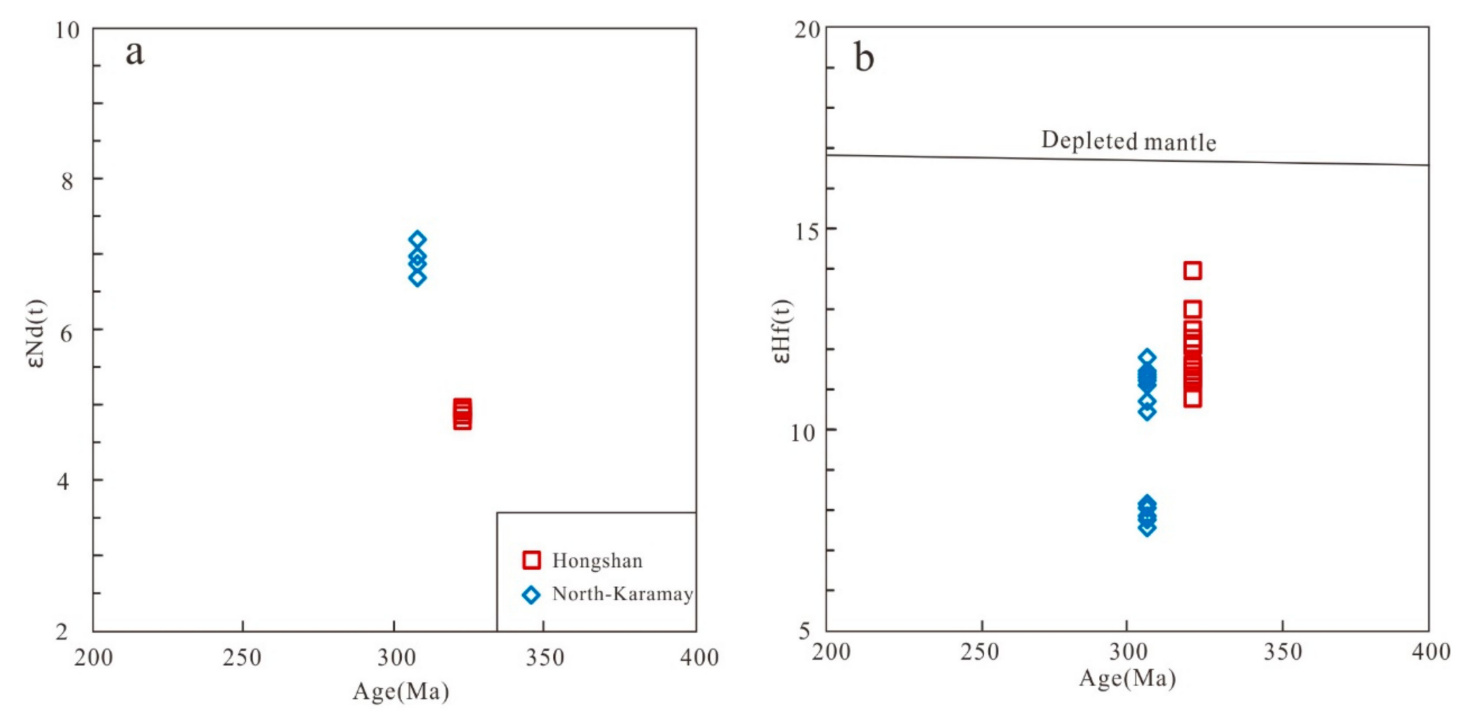

Figure 8. Plot of (a) Age- $\varepsilon N d(t)$ and $(\mathbf{b})$ Age- $\varepsilon H f(t)$ diagram for the studied pluton.

\section{Discussion}

\subsection{Magmatism in the Western Junggar}

The zircon U-Pb data for the Hongshan and North Karamay plutons presented in this study suggest that the two intrusions were emplaced at $308 \pm 5 \mathrm{Ma}$ and $323 \pm 3 \mathrm{Ma}$, respectively. Previous studied have shown that granitic intrusions in the Western Junggar were mostly emplaced between the Late Silurian and Middle Permian $[40,41,45,46,84-86]$. Three 'pulses' of granitic magmatism have been identified: 1) from 316 to $283 \mathrm{Ma}$ [13,40-42,86]; 2) from 346 to $321 \mathrm{Ma}[40,41,86]$, and; 3) from 422 to $405 \mathrm{Ma}$ [45].

The youngest plutons, which are widespread in both the northern and southern parts of the Western Junggar, consist mainly of A-type granites and adakites, with some charnockites and magnesian dikes $[4,13,30,42-44,84-86]$. The plutons in the southern region of the Western Junggar were emplaced earlier (316-287 Ma) than those in the north (303-283Ma). Late Carboniferous intrusions occured mainly in the Karamay island arc, and consist of A-type granites, I-type granites, and adakitic granites [44]. 
Table 4. Sr-Nd isotopic compositions of the Hongshan and North-Karamay granitoids.

\begin{tabular}{|c|c|c|c|c|c|c|c|c|c|c|c|c|}
\hline Sample & ${ }^{87} \mathrm{Rb} /{ }^{86} \mathrm{Sr}$ & ${ }^{87} \mathrm{Sr} /{ }^{86} \mathrm{Sr}$ & $2 \sigma$ & $\left({ }^{87} \mathrm{Sr} /{ }^{86} \mathrm{Sr}\right)_{\mathrm{i}}$ & ${ }^{147} \mathrm{Sm} /{ }^{144} \mathrm{Nd}$ & ${ }^{143} \mathrm{Nd} /{ }^{144} \mathrm{Nd}$ & $2 \sigma$ & $\left({ }^{143} \mathrm{Nd} /{ }^{144} \mathrm{Nd}\right)_{i}$ & $\mathrm{f}_{\mathrm{Sm} / \mathrm{Nd}}$ & $\varepsilon N d(t)$ & $\mathrm{T}_{\mathrm{DM1}}(\mathrm{Ma})$ & $\mathrm{T}_{\mathrm{DM} 2}(\mathrm{Ma})$ \\
\hline HS-01 & 4.350 & 0.721765 & 2 & 0.705055 & 0.1394 & 0.512866 & 3 & 0.512574 & -0.26 & 6.70 & 583 & 490 \\
\hline HS-02 & 13.802 & 0.757996 & 4 & 0.704978 & 0.1332 & 0.512863 & 2 & 0.512584 & -0.29 & 6.88 & 544 & 477 \\
\hline HS-03 & 15.163 & 0.763008 & 3 & 0.704760 & 0.1316 & 0.512865 & 4 & 0.512590 & -0.30 & 6.98 & 530 & 470 \\
\hline HS-04 & 20.988 & 0.785883 & 5 & 0.705262 & 0.1245 & 0.512862 & 2 & 0.512601 & -0.34 & 7.21 & 493 & 454 \\
\hline NK-01 & 0.431 & 0.702923 & 3 & 0.701269 & 0.1315 & 0.512751 & 2 & 0.512462 & -0.30 & 4.89 & 741 & 628 \\
\hline NK-02 & 0.438 & 0.702613 & 3 & 0.700932 & 0.1318 & 0.512748 & 2 & 0.512459 & -0.30 & 4.82 & 749 & 633 \\
\hline NK-03 & 0.472 & 0.702725 & 4 & 0.700912 & 0.1296 & 0.512750 & 1 & 0.512465 & -0.31 & 4.95 & 726 & 624 \\
\hline NK-04 & 0.431 & 0.702846 & 2 & 0.701191 & 0.1304 & 0.512747 & 4 & 0.512461 & -0.31 & 4.86 & 738 & 630 \\
\hline
\end{tabular}

$\varepsilon \mathrm{Nd}(\mathrm{t})$ values were calculated using present-day $\left({ }^{147} \mathrm{Sm} /{ }^{144} \mathrm{Nd}\right)_{\mathrm{CHUR}}=0.1967$ and $\left({ }^{143} \mathrm{Nd} /{ }^{144} \mathrm{Nd}\right)_{\mathrm{CHUR}}=0.512638 . \mathrm{T}_{\mathrm{DM}}$ values were calculated using present-day $\left({ }^{147} \mathrm{Sm} /{ }^{144} \mathrm{Nd}\right)_{\mathrm{DM}}=0.2137$ and $\left({ }^{143} \mathrm{Nd} /{ }^{144} \mathrm{Nd}\right){ }_{\mathrm{DM}}=0.51315$.

Table 5. Hf isotopic compositions of the North-Karamay granitoids and the Hongshan granitoids.

\begin{tabular}{|c|c|c|c|c|c|c|c|c|c|}
\hline Sample Name & ${ }^{176} \mathrm{Hf} /{ }^{177} \mathrm{Hf}$ & 1s & ${ }^{176} \mathrm{Yb} /{ }^{177} \mathrm{Hf}$ & $1 \mathrm{~s}$ & ${ }^{176} \mathrm{Lu} /{ }^{177} \mathrm{Hf}$ & $1 \mathrm{~s}$ & $\varepsilon \mathbf{H f}(\mathbf{t})$ & $\mathrm{T}_{\mathrm{DM1}}(\mathrm{Ma})$ & $\mathrm{T}_{\mathrm{DM} 2}(\mathrm{Ma})$ \\
\hline NK-01 & 0.282799 & 0.000010 & 0.003157 & 0.000013 & 0.001557 & 0.000012 & 7.70 & 651.95 & 839.27 \\
\hline NK-02 & 0.282881 & 0.000012 & 0.010279 & 0.000086 & 0.001104 & 0.000010 & 10.72 & 526.83 & 647.06 \\
\hline NK-03 & 0.282812 & 0.000009 & 0.000857 & 0.000001 & 0.001506 & 0.000024 & 8.18 & 632.07 & 808.79 \\
\hline NK-04 & 0.282903 & 0.000010 & 0.010384 & 0.000010 & 0.002314 & 0.000012 & 11.24 & 512.06 & 613.87 \\
\hline NK-05 & 0.282877 & 0.000009 & 0.010834 & 0.000026 & 0.001627 & 0.000035 & 10.46 & 540.08 & 663.25 \\
\hline NK-06 & 0.282804 & 0.000014 & 0.014430 & 0.000108 & 0.001130 & 0.000001 & 7.98 & 637.08 & 821.68 \\
\hline NK-07 & 0.282902 & 0.000010 & 0.014609 & 0.000020 & 0.001345 & 0.000006 & 11.39 & 501.10 & 604.40 \\
\hline NK-08 & 0.282909 & 0.000011 & 0.011777 & 0.000118 & 0.000538 & 0.000044 & 11.80 & 480.78 & 577.79 \\
\hline NK-09 & 0.282797 & 0.000011 & 0.003129 & 0.000012 & 0.001603 & 0.000011 & 7.61 & 656.05 & 845.06 \\
\hline NK-10 & 0.282879 & 0.000013 & 0.010190 & 0.000079 & 0.001744 & 0.000009 & 10.50 & 539.31 & 660.96 \\
\hline NK-11 & 0.282810 & 0.000010 & 0.000850 & 0.000001 & 0.001630 & 0.000023 & 8.07 & 637.50 & 815.65 \\
\hline NK-12 & 0.282901 & 0.000011 & 0.010293 & 0.000009 & 0.001182 & 0.000011 & 11.40 & 499.85 & 603.65 \\
\hline NK-13 & 0.282875 & 0.000010 & 0.010740 & 0.000024 & 0.001176 & 0.000033 & 10.48 & 536.85 & 662.30 \\
\hline NK-14 & 0.282802 & 0.000015 & 0.014305 & 0.000099 & 0.001294 & 0.000001 & 7.86 & 643.17 & 829.10 \\
\hline NK-15 & 0.282899 & 0.000011 & 0.014482 & 0.000018 & 0.001337 & 0.000006 & 11.31 & 504.29 & 609.48 \\
\hline HS-01 & 0.282922 & 0.000013 & 0.037394 & 0.005785 & 0.006269 & 0.000164 & 10.79 & 543.65 & 630.70 \\
\hline HS-02 & 0.282936 & 0.000014 & 0.089796 & 0.001938 & 0.002100 & 0.000064 & 12.12 & 461.67 & 545.92 \\
\hline HS-03 & 0.282927 & 0.000012 & 0.055288 & 0.004316 & 0.004677 & 0.000129 & 11.30 & 510.09 & 598.51 \\
\hline HS-04 & 0.282904 & 0.000011 & 0.025720 & 0.000932 & 0.001010 & 0.000027 & 11.22 & 493.25 & 603.32 \\
\hline HS-05 & 0.282900 & 0.000011 & 0.036632 & 0.000383 & 0.000415 & 0.000014 & 11.21 & 490.74 & 603.96 \\
\hline
\end{tabular}


Table 5. Cont

\begin{tabular}{|c|c|c|c|c|c|c|c|c|c|}
\hline Sample Name & ${ }^{176} \mathrm{Hf} /{ }^{177} \mathrm{Hf}$ & $1 \mathrm{~s}$ & ${ }^{176} \mathrm{Yb} /{ }^{177} \mathrm{Hf}$ & $1 \mathrm{~s}$ & ${ }^{176} \mathrm{Lu} /{ }^{177} \mathrm{Hf}$ & $1 \mathrm{~s}$ & $\varepsilon H f(t)$ & $\mathrm{T}_{\mathrm{DM1}}(\mathrm{Ma})$ & $\mathrm{T}_{\mathrm{DM} 2}(\mathrm{Ma})$ \\
\hline HS-06 & 0.282940 & 0.000013 & 0.049781 & 0.001850 & 0.002005 & 0.000063 & 12.28 & 454.88 & 536.02 \\
\hline HS-07 & 0.282916 & 0.000010 & 0.055865 & 0.002550 & 0.002763 & 0.000082 & 11.30 & 499.03 & 598.23 \\
\hline HS- 08 & 0.282922 & 0.000012 & 0.080882 & 0.000751 & 0.000813 & 0.000032 & 11.90 & 465.11 & 559.86 \\
\hline HS-09 & 0.282918 & 0.000011 & 0.060288 & 0.002136 & 0.002314 & 0.000066 & 11.46 & 490.18 & 588.30 \\
\hline HS-10 & 0.282921 & 0.000011 & 0.031263 & 0.002120 & 0.002298 & 0.000064 & 11.57 & 485.34 & 580.99 \\
\hline HS-11 & 0.282916 & 0.000010 & 0.019363 & 0.002198 & 0.002382 & 0.000066 & 11.35 & 495.05 & 595.23 \\
\hline HS-12 & 0.282934 & 0.000013 & 0.026723 & 0.001571 & 0.001703 & 0.000053 & 12.15 & 459.01 & 544.37 \\
\hline HS-13 & 0.282980 & 0.000012 & 0.087979 & 0.000698 & 0.000756 & 0.000056 & 13.96 & 383.01 & 428.75 \\
\hline HS-14 & 0.282944 & 0.000012 & 0.025627 & 0.001611 & 0.001746 & 0.000054 & 12.49 & 445.13 & 522.45 \\
\hline HS-15 & 0.282960 & 0.000012 & 0.031248 & 0.001811 & 0.001963 & 0.000024 & 13.00 & 425.09 & 490.11 \\
\hline
\end{tabular}

The ${ }^{176} \mathrm{Hf} /{ }^{177} \mathrm{Hf}$ and ${ }^{176} \mathrm{Lu} /{ }^{177} \mathrm{Hf}$ ratios of chondrite and depleted mantle at the present are 0.282772 and $0.0332,0.28325$ and $0.0384 ;\left({ }^{176} \mathrm{Lu} /{ }^{177} \mathrm{Hf}\right){ }_{\mathrm{LC}}=0.019 ; \lambda=1.867 \times 10^{-11}$ year ${ }^{-1}$; $\mathrm{t}=$ crystallization time of zircon. $\left.\varepsilon \mathrm{Hf}(\mathrm{t})=\left\{\left[\left[{ }^{176} \mathrm{Hf} /{ }^{177} \mathrm{Hf}\right)_{\mathrm{S}}-\left({ }^{176} \mathrm{Lu} /{ }^{177} \mathrm{Hf}\right)_{S} \times(\mathrm{e} \lambda \mathrm{t}-1)\right] /\left[{ }^{176} \mathrm{Hf} /{ }^{177} \mathrm{Hf}\right)_{\mathrm{CHUR}}-\left({ }^{176} \mathrm{Lu} /{ }^{177} \mathrm{Hf}\right)_{\mathrm{CHUR}} \times(\mathrm{e} \lambda \mathrm{t}-1)\right]-1\right\} \times 10000 . \mathrm{T}_{\mathrm{DM} 1}=1 / \lambda \ln \left\{\left[\left({ }^{176} \mathrm{Hf} /{ }^{177} \mathrm{Hf}\right)_{S}-\right.\right.$ $\left.\left.\left({ }^{176} \mathrm{Hf} /{ }^{177} \mathrm{Hf}\right)_{\mathrm{DM}}\right] /\left[\left({ }^{176} \mathrm{Lu} /{ }^{177} \mathrm{Hf}\right)_{\mathrm{S}}-\left({ }^{176} \mathrm{Lu} /{ }^{177} \mathrm{Hf}\right)_{\mathrm{DM}}\right]+1\right\} . \mathrm{T}_{\mathrm{DM} 2}=\mathrm{t}+1 / \lambda \ln \left\{\left[\left({ }^{176} \mathrm{Hf} /{ }^{177} \mathrm{Hf}\right)_{S}-\left({ }^{176} \mathrm{Hf} /{ }^{177} \mathrm{Hf}\right)_{\mathrm{DM}}\right] /\left[\left({ }^{176} \mathrm{Lu} /{ }^{177} \mathrm{Hf}\right)_{\mathrm{LC}}-\left({ }^{176} \mathrm{Lu} /{ }^{177} \mathrm{Hf}\right)_{\mathrm{DM}}\right]+1\right\}$. 


\subsection{Petrogenesis and Magma Source}

\subsubsection{North Karamay Granitoids}

All of the North Karamay granitoids plot in the adakite field on the $\mathrm{Sr} / \mathrm{Y}$ vs. $\mathrm{Y}$ and $(\mathrm{La} / \mathrm{Yb})_{\mathrm{N}} \mathrm{vs}$. $(\mathrm{Yb})_{\mathrm{N}}$ discrimination diagrams (Figure 9a,b; [78,87]), indicating an adakitic affinity. The genesis of adakitic magmas remains the subject of debate, and several major hypotheses have been proposed, including (1) assimilation and low-pressure fractional crystallization in basaltic parent magmas [88], (2) partial melting of thickened lower crust (high $\mathrm{SiO}_{2}$ and $\mathrm{K}_{2} \mathrm{O}$ contents and low $\mathrm{MgO}, \mathrm{Cr}$, and Ni contents, [89-92]), (3) partial melting of delaminated lower crust (high $\mathrm{Cr}$, Co and Ni contents; [93-96]), (4) mixing of basaltic and adakitic magmas [97], (5) partial melting of young, hot, recently subducted oceanic crust [98], and (6) melting of subducted continental crust [99].
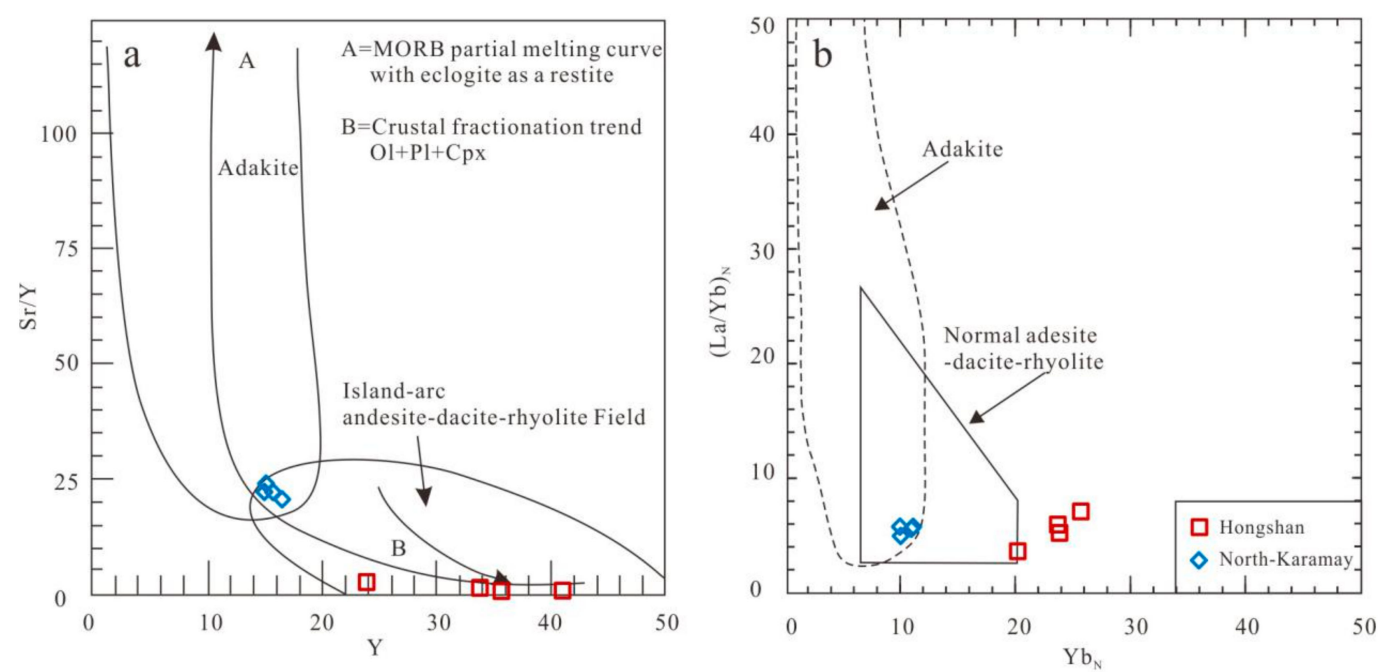

Figure 9. (a) Sr/Y vs. Y (after Defant et al. [79]), and (b) $(\mathrm{La} / \mathrm{Yb})_{\mathrm{N}}$ vs. $(\mathrm{Yb})_{\mathrm{N}}$ diagrams (Geng et al. [13]).

The studied adakitic granodiorites show none of the compositional trends characteristic of low-pressure or high-pressure assimilation fractional crystallization (AFC) (Figure 10), and it is therefore unlikely that they represent the product of low-pressure or high-pressure fractional crystallization. The North Karamay granitoids have very low $\mathrm{Mg}^{\#}$ values $(0.37-0.38), \mathrm{Cr}(<16.0 \mathrm{ppm})$, $\mathrm{Co}(<8.1 \mathrm{ppm})$ and $\mathrm{Ni}(<6.6 \mathrm{ppm})$ content, meaning they are unlikely to have been formed by melt-mantle interaction, which usually occurs when delaminated lower crust melt interacts with mantle peridotite (Figure 11a-c; [93,94]). The studied granitoids do show highly positive $\varepsilon \mathrm{Nd}(\mathrm{t})$ and $\varepsilon H f(t)$ values, which are distinctly different from those of adakites formed via partial melting of continental crust; in Tibet, these typically show low, negative $\varepsilon \mathrm{Nd}(\mathrm{t})$ and $\varepsilon \mathrm{Hf}(\mathrm{t})$ values [99]. 

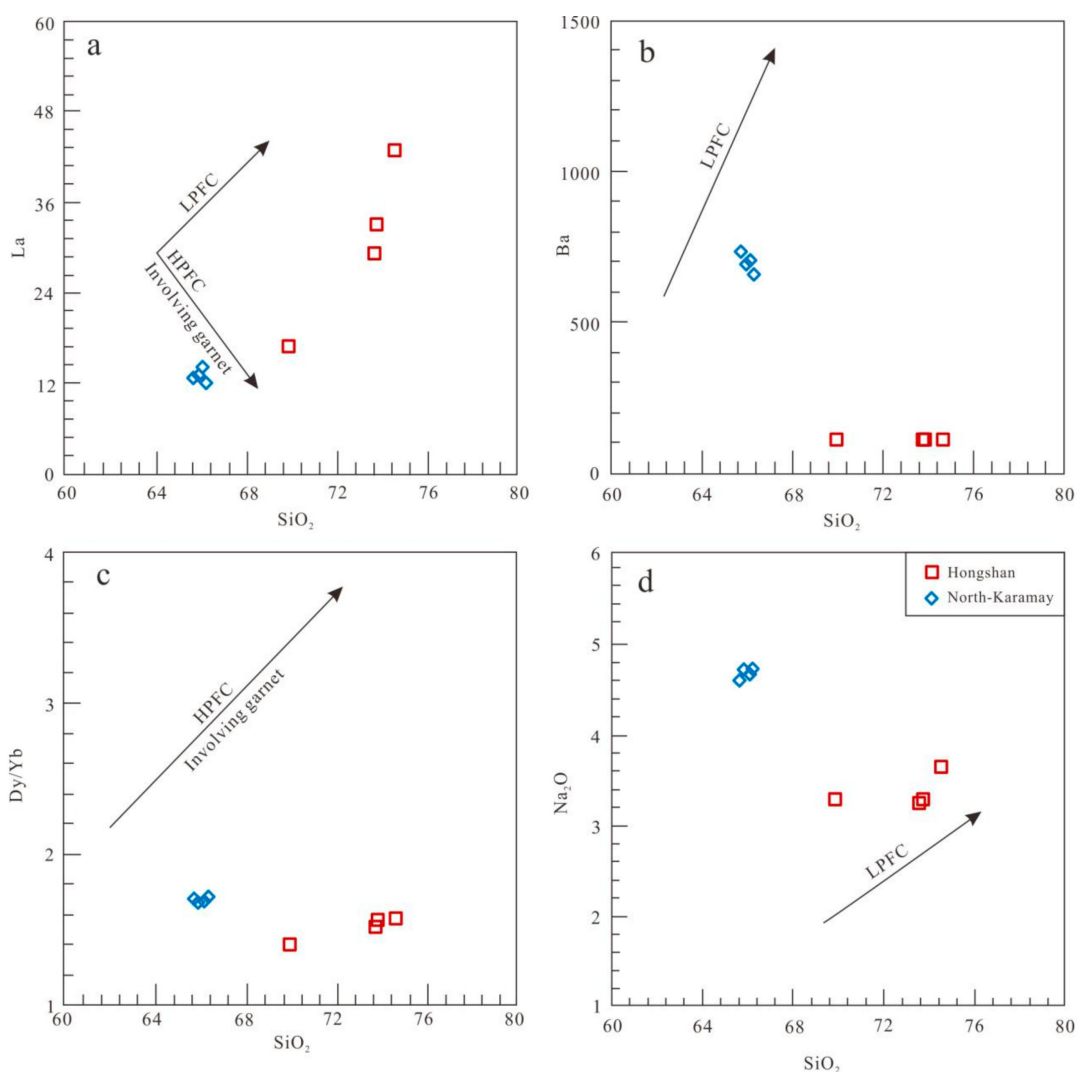

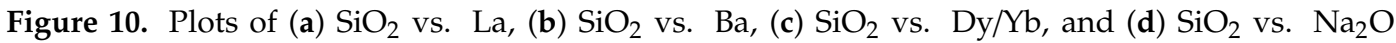
diagram. Fractional crystallization trends in $(\mathrm{a}-\mathrm{d})$ : HPFC, high-pressure fractional crystallization involving garnet [100]; LPFC, low-pressure fractional crystallization involving olivine + clinopyroxene + plagioclase + hornblende + titanomagnetite [88].
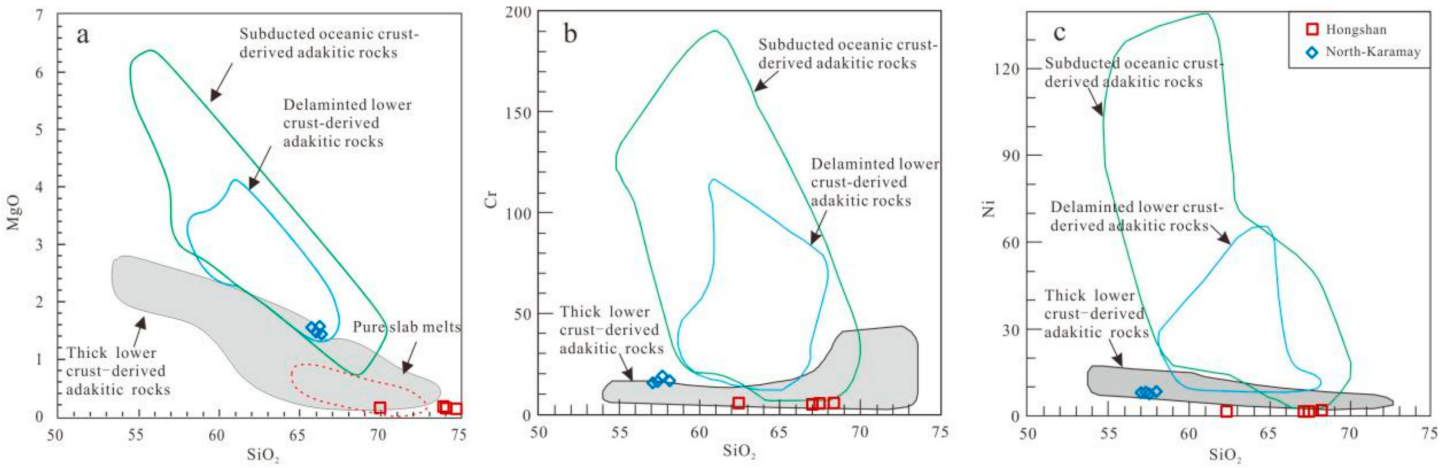

Figure 11. Plots of (a) $\mathrm{SiO}_{2}$ vs. $\mathrm{MgO}$, (b) $\mathrm{SiO}_{2}$ vs. Cr diagram, and (c) $\mathrm{SiO}_{2}$ vs. Ni diagram [96]

No mafic enclaves have been observed in the adakitic granites, suggesting that they did not form via mixing of basaltic and adakitic magmas. The adakitic granodiorites also have lower $\varepsilon \mathrm{Nd}(\mathrm{t})$ values $(+4.82$ to +4.95$)$ than the A-type granites $(+6.70$ to +7.21$)$ (Figure $8 \mathrm{a})$, which represent the most crust-derived, felsic end member in the studied region. This rules out the possibility that the adakitic granodiorites are the product of mixing between basaltic and adakitic magmas. Therefore, we suggest that the adakitic granodiorites were most likely generated by partial melting of the lower crust. This scenario explains both the relatively high $\mathrm{SiO}_{2}$ and $\mathrm{K}_{2} \mathrm{O}$ content, and the low $\mathrm{MgO}, \mathrm{Cr}$, and Ni content.

\subsubsection{Hongshan Granitoids}

The Hongshan granitoids are characterized by high alkaline content, as well as high $\mathrm{Fe}, \mathrm{Zr}$, and $\mathrm{Nb}$ content. They display prominent negative anomalies in $\mathrm{Ba}, \mathrm{Sr}, \mathrm{P}, \mathrm{Eu}$, and $\mathrm{Ti}$ (Figure $6 \mathrm{a}, \mathrm{b}$ ), and 
high $10^{4} \mathrm{Ga} / \mathrm{Al}$ ratios, similar to typical A-type granites [101,102]. All samples from the Hongshan plutons plot in the A-type field on the $10^{4} \mathrm{Ga} / \mathrm{Al}$ vs. $\mathrm{Zr}$ and $10^{4} \mathrm{Ga} / \mathrm{Al}$ vs. $\left(\mathrm{K}_{2} \mathrm{O}+\mathrm{Na}_{2} \mathrm{O}\right)$ discrimination diagrams (Figure 12a,b; [101]).
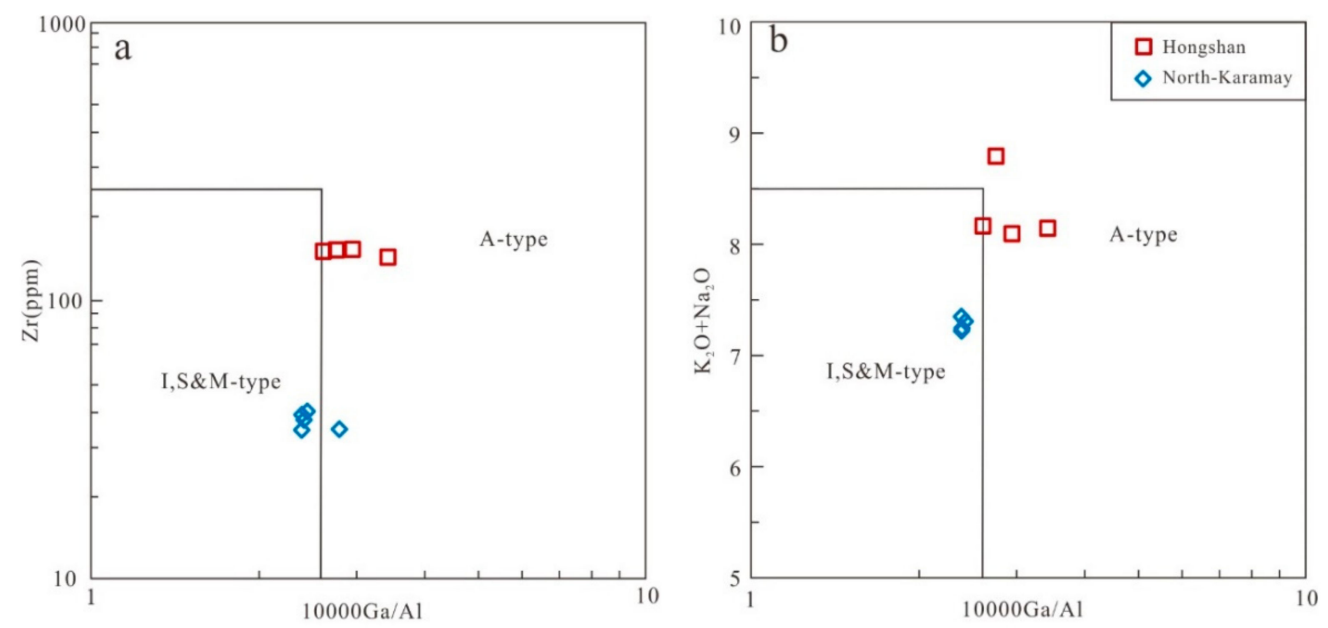

Figure 12. Trace element discrimination diagrams of the studied granitoids for rock classification: (a) $10000 \mathrm{Ga} / \mathrm{Al}$ vs. $\mathrm{Zr}$ and (b) $10000 \mathrm{Ga} / \mathrm{Al}$ vs. $\left(\mathrm{K}_{2} \mathrm{O}+\mathrm{Na}_{2} \mathrm{O}\right)$ diagrams [101].

The depleted Nd-Hf isotopic compositions of the Hongshan granitoids $(\varepsilon \mathrm{Nd}(\mathrm{t})=+6.70$ to +7.21 ; $\varepsilon \mathrm{Hf}(\mathrm{t})=+10.79$ to +13.00 , Tables 4 and 5) imply that they are unlikely to have been generated via partial melting of crystalline basement rocks, which typically have distinctly negative $\varepsilon \mathrm{Nd}(\mathrm{t})$ values in the Western Junggar [103]. Other potential Carboniferous sources in the Western Junggar region include Early Carboniferous arc-type basalts and basaltic andesites [55] and the Late Carboniferous Hatu tholeiitic basalts [104]. However, the Sr and $\mathrm{Nd}$ isotope ratios of the Hongshan granites are much greater than those of the contemporary Hatu basalts (Figure 13a). Therefore, the Hongshan A-type granites could not have been generated via fractional crystallization of mantle-derived arc basalt melts.
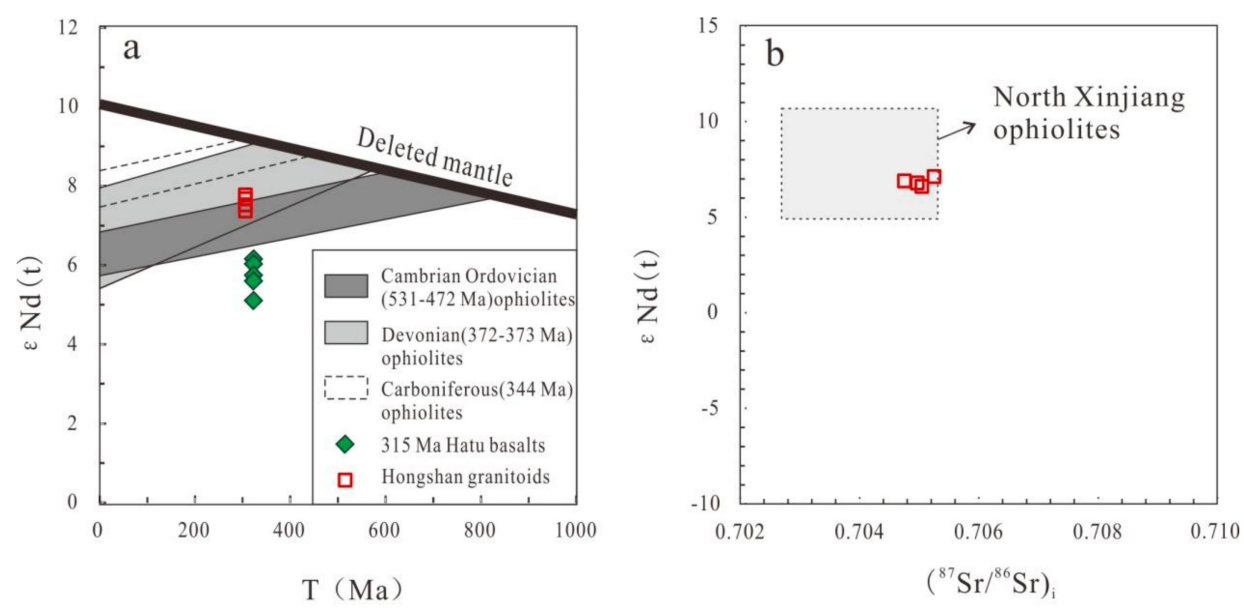

Figure 13. Plot of Sr-Nd isotopic ratios of the Hongshan A-type granites: (a) Age- $\varepsilon \mathrm{Nd}(\mathrm{t})$, and

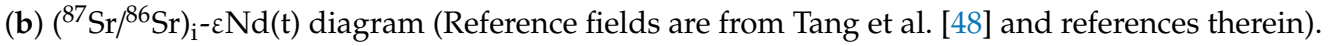

These data suggest that these A-type granites likely formed via partial melting of the oceanic crust, based on the following lines of evidence: (1) the negative Eu anomalies and high concentrations of HREEs; (2) the field encompassing the Hongshan A-type granites partially overlies that of the North Xinjiang ophiolites (Figure 13a,b). Therefore, we proposed that the Hongshan A-type granites are derived from partial melting of the oceanic crust. 
Previous studies have demonstrated that partial melting of oceanic crustal components stored in the lower crust could provide the parent magma for A-type granites [48]. The lower and middle crust of the Western Junggar intra-oceanic arc would likely have consisted of the arc itself, sitting on top of basaltic oceanic crust [20]; it may also have included underthrust or accreted oceanic crustal components $[105,106]$. Therefore, we propose here that the A-type granites are mainly derived from melting of the lower and middle crust of an intra-oceanic arc, which largely consisted of oceanic crust.

\subsection{Tectonic Implications}

All of the studied granitoids plot in the "VAG" field on the $\mathrm{Rb} v s$. ( $\mathrm{Yb}+\mathrm{Ta}$ ) and $\mathrm{Rb}$ vs. $(\mathrm{Y}+\mathrm{Nb}$ ) tectonic discrimination diagrams (Figure 14a,b), indicating that they are likely to have been formed during island-arc magmatism. Our zircon U-Pb data indicate a Late Carboniferous (ca. 322-307 Ma) magmatic "flare up" occurred in the Western Junggar. Asthenospheric sources of heat are required to explain the formation of the studied granites, and two geodynamic models have been proposed: subduction of an oceanic ridge $[13,47,48,107]$, or the rollback of a subducting slab $[85,86]$. Both of these processes are capable of generating adakites and A-type granites in a subduction setting [12,48,98,108-112].
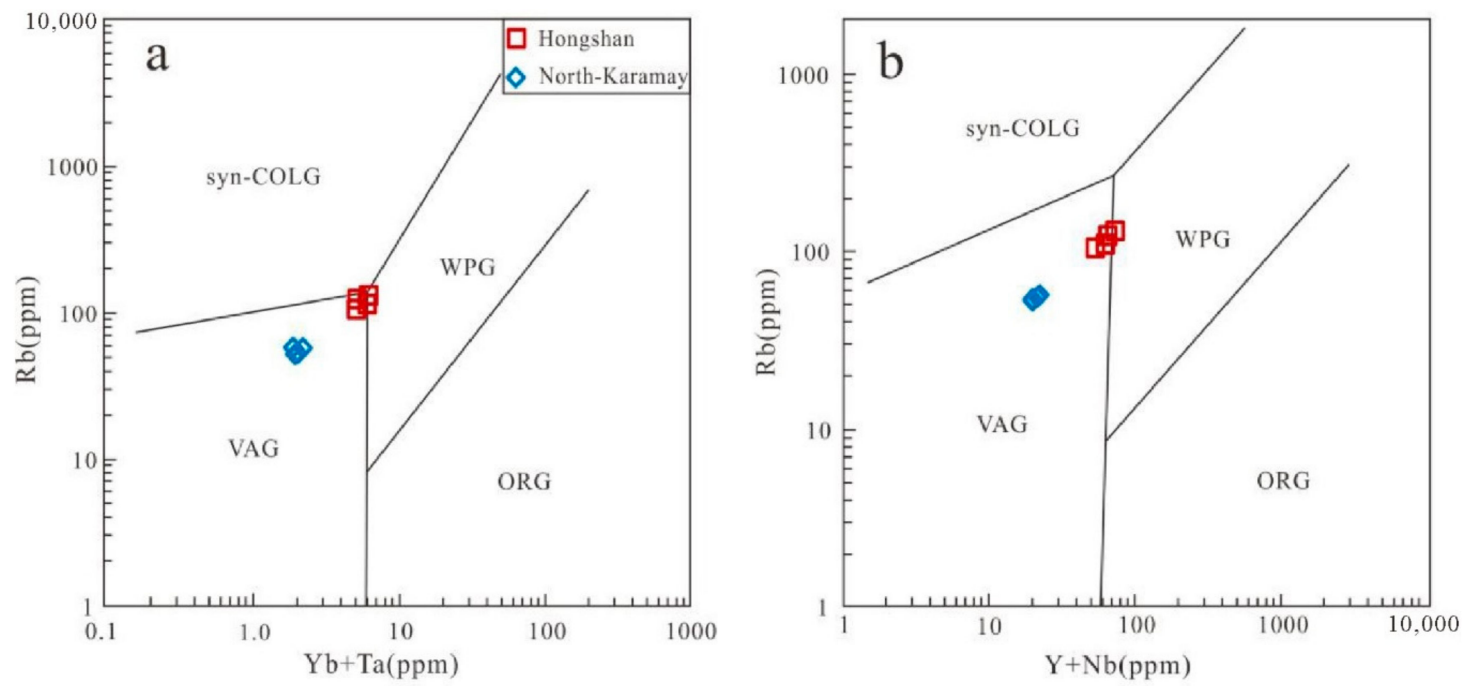

Figure 14. Tectonic discrimination diagramwith trace elemental plots of the studied granitoids: (a) $\mathrm{Rb}$ vs. $(\mathrm{Yb}+\mathrm{Ta})$ (Pearce et al. [112]), and (b) Rb vs. $(\mathrm{Y}+\mathrm{Nb})$ (Pearce et al. [112]), tectonic discrimination diagrams.

If this ridge subduction model is accurate, the distribution of adakites and A-type granites would imply a spreading ridge oriented sub-parallel to the subduction trench, and perpendicular to the suture zone. However, The Devonian-Carboniferous granitoids in the Karamay region define a narrow, linear zone of magmatism parallel to the subduction zone, which is inconsistent with a ridge subduction model. In addition, the adakitic rocks in a slab window are typically characterized by high $\mathrm{Cr}, \mathrm{Co}$, and $\mathrm{Ni}$ content [85], whereas the Karamay adakitic granites show generally low $\mathrm{Cr}, \mathrm{Co}$, and $\mathrm{Ni}$ content. Moreover, the adakitic granodiorites and A-type granites are almost contemporaneous with arc volcanic rocks, which does not support the ridge subduction model [86].

An alternative explanation is that asthenospheric upwelling resulted from the rollback of a subducting slab of Junggar oceanic crust. This scenario would also have provided the required high heat flow to drive partial melting of the subducting crust (Figure 15). In this model, the lithosphere is negatively buoyant, causing the subducting slab to sink vertically $[113,114]$. If the slab sinks into the mantle faster than it converges with the overriding plate, the subduction trench is expected to 'roll back' from the overriding plate [115]. Slab rollback could induce upwelling of the asthenosphere to compensate for the loss in volume of the mantle wedge $[86,116]$. 


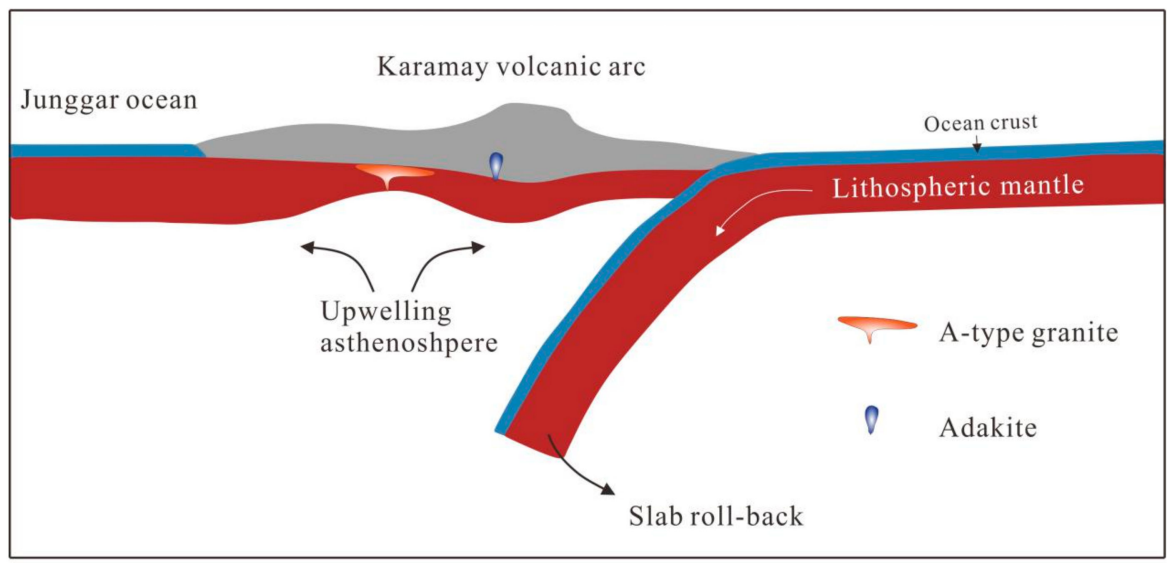

Figure 15. Tectonic model for slab roll-back to explain the generation of studied granitoids from Western Junggar.

During the Late Silurian to Early Devonian, the oceanic crust underlying the Junggar Ocean was being consumed beneath the Karamay volcanic arc. With ongoing extension during the rollback of the Junggar slab, the crust and lithospheric mantle became progressively thinner. Upwelling of the asthenosphere might have triggered partial melting of the oceanic crust and overlying lower and middle crustal rocks, generating adakitic magmas and A-type granitic magmas [117,118]. Therefore, we conclude that slab rollback is the most likely explanation for the Late Silurian to Early Devonian magmatic "flare up" in the Western Junggar.

\section{Conclusions}

1. The two studied granitoids from the Western Junggar can be classified as an adakitic granodiorite and an A-type granite. Both were emplaced in the period from 322-307 Ma.

2. The adakitic granodiorites were likely generated from the partial melting of thickened lower crust. The A-type granites may have formed from partial melting of lower and middle crust in an intra-oceanic arc setting, where the melted material mainly consisted of oceanic crust.

3. Slab rollback appears to have played an important role in the generation of arc-related igneous rocks, and in the continental growth of the Central Asian Orogenic Belt.

Author Contributions: Conceptualization, J.L. and C.Z.; methodology, J.L. and D.L.; investigation, C.Z. and D.L.; writing-original draft preparation, J.L. and C.Z.; writing-review and editing, D.L. All authors have read and agreed to the published version of the manuscript.

Funding: This research was funded by the National Natural Science Foundation of China, grant number 41502209 and the National Science and Technology Major Project, grant number 2016ZX05034-001 and 2017ZX05035-002.

Acknowledgments: Jia $\mathrm{Lu}$ and Chen Zhang contributed equally to this work. We are very grateful to two anonymous reviewers for their peer-reviews and constructive comments that significantly improved the manuscript.

Conflicts of Interest: The authors declare no conflict of interest.

\section{References}

1. Hofmann, A.W. Mantle geochemistry: The message from oceanic volcanism. Nature 1997, 385, $219-229$. [CrossRef]

2. Wang, H.C.; Zhao, F.Q.; Li, H.M.; Sun, L.X.; Miao, L.C.; Ji, S.P. Zircon shrimp U-Pb age of the dioritic rocks from northern hebei: The geological records of late paleozoic magmatic arc. Acta Petrol. Sin. 2007, 23, 597-604.

3. König, S.; Münker, C.; Schuth, S.; Garbeschönberg, D. The geochemical behaviour of Sb, Mo and W in subduction zones. Geochim. Cosmochim. Acta 2008, 72, A488. 
4. Chen, B.; Arakawa, Y. Elemental and Nd-Sr isotopic geochemistry of granitoids from the West Junggar Foldbelt (NW China), with implications for phanerozoic continental growth. Geochim. Cosmochim. Acta 2005, 69, 1307-1320. [CrossRef]

5. Jahn, B.M. The central asian orogenic belt and growth of the continental crust in the phanerozoic. Geo. Soc. Lond. Spec. Pub. 2004, 226, 73-100. [CrossRef]

6. Kay, S.M.; Mpodozis, C. Central andean ore deposits linked to evolving shallow subduction systems and thickening crust. GSA Today 2001, 11, 4-9. [CrossRef]

7. Ramos, V.A.; Folguera, A. Andean flat-slab subduction through time. Geol. Soc. Lond. Spec. Pub. 2009, 327, 31-54. [CrossRef]

8. Guivel, C.; Morata, D.; Pelleter, E.; Espinoza, F.; Maury, R.C.; Lagabrielle, Y. Miocene to late quaternary patagonian basalts $\left(46-47^{\circ} \mathrm{S}\right)$ : Geochronometric and geochemical evidence for slab tearing due to active spreading ridge subduction. J. Volcanol. Geotherm. Res. 2006, 149, 346-370. [CrossRef]

9. Pallares, C.; Maury, R.C.; Bellon, H.; Royer, J.Y.; Calmus, T.; Aguillón-Robles, A. Slab-tearing following ridge-trench collision: Evidence from miocene volcanism in baja california, méxico. J. Volcanol. Geotherm. Res. 2007, 161, 95-117. [CrossRef]

10. Von Blanckenburg, F.; Davies, J.H. Slab breakoff: A model for syncollisional magmatism and tectonics in the alps. Tectonics 1995, 14, 120-131. [CrossRef]

11. Abratis, M.; Wörner, G. Ridge collision, slab-window formation, and the flux of pacific asthenosphere into the Caribbean realm. Geology 2001, 29, 127-130. [CrossRef]

12. Guivel, C.; Lagabrielle, Y.; Bourgois, J.; Martin, H.; Arnaud, N.; Fourcade, S. Very shallow melting of oceanic crust during spreading ridge subduction: Origin of near-trench quaternary volcanism at the Chile triple junction. J. Geophys. Res. Solid Earth. 2003, 108, 457-470. [CrossRef]

13. Geng, H.Y.; Sun, M.; Yuan, C.; Xiao, W.; Xian, W.; Zhao, G. Geochemical, sr-nd and zircon U-Pb-Hf isotopic studies of Late Carboniferous magmatism in the West Junggar, Xinjiang: Implications for ridge subduction? Chem. Geol. 2009, 266, 364-389. [CrossRef]

14. Xiao, W.; Santosh, M. The western central Asian orogenic belt: A window to accretionary orogenesis and continental growth. Gondwana Res. 2014, 25, 1429-1444. [CrossRef]

15. Kurasawa, H.; Konda, T. Strontium isotopic ratios of Tertiary volcanic rocks of northeastern Honshu, Japan: Implication for the spreading of the Japan Sea. J. Geol. Soc. Japan 1986, 92, 205-217. [CrossRef]

16. Arai, H.; Yamamoto, A.; Matsuzawa, Y.; Saito, Y.; Yamada, N.; Oikawa, S. Polymorphisms of apolipoprotein e and methylenetetrahydrofolate reductase in the Japanese population. J. Atheroscler. Thromb. 2007, 14, 167. [CrossRef]

17. Nohda, S.; Tatsumi, Y.; Otofuji, Y.I.; Matsuda, T.; Ishizaka, K. Asthenospheric injection and back-arc opening: Isotopic evidence from northeast Japan. Chem. Geol. 1988, 68, 317-327. [CrossRef]

18. Tatsumi, Y.; Nohda, S.; Ishizaka, K. Secular variation of magma source compositions beneath the Northeast Japan arc. Chem. Geol. 1988, 68, 309-316. [CrossRef]

19. Chin, C.D.; Linder, V.; Sia, S.K. Commercialization of microfluidic point-of-care diagnostic devices. Lab Chip. 2012, 12, 2118-2134. [CrossRef]

20. Şengör, A.M.C. Some current problems on the tectonic evolution of the Mediterranean during the Cainozoic. NATO ASI 1993, 402, 1-51.

21. Şengör, A.M.C.; Natal_in, B.A. Turkic-type orogeny and its role in the making of the continental crust. Annu. Rev. Earth Planet. Sci. 1996, 24, 263-337. [CrossRef]

22. Yakubchuk, A. Architecture and mineral deposit settings of the altaid orogenic collage: A revised model. J. Asian Earth Sci. 2004, 23, 761-779. [CrossRef]

23. Xiao, W.; Han, C.; Chao, Y.; Min, S.; Lin, S.; Chen, H. Middle Cambrian to Permian subduction-related accretionary orogenesis of Northern Xinjiang, NW China: Implications for the tectonic evolution of central Asia. J. Asian Earth Sci. 2008, 32, 102-117. [CrossRef]

24. Xiao, W.; Huang, B.; Han, C.; Shu, S.; Li, J. A review of the western part of the altaids: A key to understanding the architecture of accretionary orogens. Gondwana Res. 2010, 18, 253-273. [CrossRef]

25. Xiao, W.; Sun, M.; Santosh, M. Continental reconstruction and metallogeny of the Circum-Junggar areas and termination of the southern Central Asian Orogenic Belt. Geosci. Front. 2015, 6, 137-140. [CrossRef]

26. Zhang, C.; Liu, D.D.; Zeng, J.H.; Jiang, S.; Luo, Q.; Kong, X.Y.; Yang, W.; Liu, L.F. Nd-O-Hf isotopic decoupling in S-type granites: Implications for ridge subduction. Lithos 2019, 332-333, 261-273. [CrossRef] 
27. Zhang, C.; Jiang, S.; Liu, D.D.; Chakrabarti, R.; Zeng, J.H.; Santosh, M.; Luo, Q.; Spencer, C.J.; Ma, C.; Liu, L.F.; et al. A novel model for silicon recycling in the lithosphere: Evidence from the Central Asian Orogenic Belt. Gondwana Res. 2019, 76, 115-122. [CrossRef]

28. Zhang, C.; Liu, D.; Zhang, X.; Spencer, C.; Tang, M.; Zeng, J.; Jiang, S.; Jolivet, M.; Kong, X. Hafnium isotopic disequilibrium during sediment melting and assimilation. Geochem. Persp. Let. 2020, 12, 34-39. [CrossRef]

29. Zhang, C.; Zhang, X.; Santosh, M.; Liu, D.D.; Ma, C.; Zeng, J.H.; Jiang, S.; Luo, Q.; Kong, X.Y.; Liu, L.F. Zircon Hf-O-Li isotopes of granitoids from the Central Asian Orogenic Belt: Implications for supercontinent evolution. Gondwana Res. 2020, 83, 132-140. [CrossRef]

30. Chen, B.; Jahn, B.M. Genesis of post-collisional granitoids and basement nature of the Junggar terrane, NW China: Nd-Sr isotope and trace element evidence. J. Asian Earth Sci. 2004, 23, 691-703. [CrossRef]

31. Windley, B. Chapter 1.1 overview and history of investigation of early earth rocks. Dev. Precambrian Geol. 2007, 15, 3-7.

32. Xiao, W.J.; Windley, B.F.; Huang, B.C.; Han, C.M.; Yuan, C.; Chen, H.L.; Sun, M.; Li, J.L. End-Permian to mid-Triassic termination of the accretionary processes of the southern Altaids: Implications for the geodynamic evolution, Phanerozoic continental growth, and metallogeny of Central Asia. Int. J. Earth Sci. (Geol. Rundsch.) 2009, 98, 1189-1217. [CrossRef]

33. Biske, Y.S.; Seltmann, R. Paleozoic Tian-shan as a transitional region between the rheic and urals-turkestan oceans. Gondwana Res. 2010, 17, 602-613. [CrossRef]

34. Han, B.F.; Wang, S.G.; Jahn, B.M.; Hong, D.W.; Kagami, H.; Sun, Y.L. Depleted-mantle source for the ulungur river a-type granites from North Xinjiang, China: Geochemistry and nd-sr isotopic evidence, and implications for phanerozoic crustal growth. Geol. Chem. Miner. 1997, 138, 135-159. [CrossRef]

35. Jahn, B.M.; Wu, F.Y.; Chen, B. Granitoids of the central asian orogenic belt and continental growth in the phanerozoic. Trans. R. Soc Edinb. Earth 2000, 91, 181-193. [CrossRef]

36. Yuan, C.; Sun, M.; Xiao, W.; Li, X.; Chen, H.; Lin, S. Accretionary orogenesis of the Chinese Altai: Insights from Paleozoic granitoids. Chem. Geol. 2007, 242, 22-39. [CrossRef]

37. Zhang, C.; Santosh, M.; Liu, L.F.; Luo, Q.; Zhang, X.; Liu, D.D. Early Silurian to Early Carboniferous ridge subduction in NW Junggar: Evidence from geochronological, geochemical, and Sr-Nd-Hf isotopic data on alkali granites and adakites. Lithos 2018, 300-301, 314-329. [CrossRef]

38. Zhang, C.; Luo, Q.; Zhang, X.; Liu, L.F.; Liu, D.D.; Wang, P.F.; Yang, K.J.; Wang, J.; Zhao, Y. Geochronological, geochemical, and $\mathrm{Sr}-\mathrm{Nd}-\mathrm{Hf}$ isotopic studies of the Aketas adakitic granites in Eastern Junggar: Petrogenesis and tectonic implications. Geol. J. 2018, 5, 80-101. [CrossRef]

39. Zhang, C.; Liu, D.D.; Luo, Q.; Liu, L.F.; Zhang, Y.Z.; Zhu, D.Y.; Wang, P.F.; Dai, Q.Q. An evolving tectonic environment of Late Carboniferous to Early Permian granitic plutons in the Chinese Altai and Eastern Junggar terranes, Central Asian Orogenic Belt, NW China. J. Asian Earth Sci. 2017, 159, 185-208. [CrossRef]

40. Zhang, C.; Liu, L.F.; Santosh, M.; Luo, Q.; Zhang, X. Sediment recycling and crustal growth in the Central Asian Orogenic Belt: Evidence from Sr-Nd-Hf isotopes and trace elements in granitoids of the Chinese Altay. Gondwana Res. 2017, 47, 142-160. [CrossRef]

41. Tang, D.M.; Qin, K.Z.; Sun, H.; Su, B.X.; Xiao, Q.H. The role of crustal contamination in the formation of Ni-Cu sulfide deposits in eastern tianshan, xinjiang, northwest China: Evidence from trace element geochemistry, Re-Os, Sr-Nd, zircon Hf-O, and sulfur isotopes. J. Asian Earth Sci. 2012, 49, 145-160. [CrossRef]

42. Yang, G.; Li, Y.; Safonova, I.; Yi, S.; Tong, L.; Seltmann, R. Early carboniferous volcanic rocks of west Junggar in the western central asian orogenic belt: Implications for a supra-subduction system. Int. Geol. Rev. 2014, 56, 823-844. [CrossRef]

43. Feng, Z.Z.; Chen, J.X.; Sheng, H. Lithofacies paleogeography of Early Paleozoic of North China Platform. Acta Sedimentol. Sin. 1989, 7, 16-55.

44. Han, B.F.; Ji, J.Q.; Song, B.; Chen, L.H. Late paleozoic vertical growth of continental crust around the Junggar basin, Xinjiang, China (part I): Timing of post-collisional plutonism. Acta Petrol. Sin. 2006, 22, 1077-1086.

45. Zhou, T.; Yuan, F.; Fan, Y.; Zhang, D.; Cooke, D.; Zhao, G. Granites in the Sawuer region of the West Junggar, Xinjiang Province, China: Geochronological and geochemical characteristics and their geodynamic significance. Lithos 2008, 106, 191-206. [CrossRef]

46. Tang, G.J.; Wang, Q.; Wyman, D.A.; Sun, M.; Li, Z.X.; Zhao, Z.H. Geochronology and geochemistry of late paleozoic magmatic rocks in the lamasu-dabate area, Northwestern Tianshan (West China): Evidence for a tectonic transition from arc to post-collisional setting. Lithos 2010, 119, 393-411. [CrossRef] 
47. Tang, G.J.; Wang, Q.; Wyman, D.A.; Li, Z.X.; Zhao, Z.H.; Yang, Y.H. Late Carboniferous high $\varepsilon N d(t)-\varepsilon H f(t)$ granitoids, enclaves and dikes in Western Junggar, NW China: Ridge-subduction-related magmatism and crustal growth. Lithos 2012, 140-141, 86-102. [CrossRef]

48. Tang, G.J.; Wang, Q.; Wyman, D.A.; Li, Z.X.; Xu, Y.G.; Zhao, Z.H. Recycling oceanic crust for continental crustal growth: Sr-Nd-Hf isotope evidence from granitoids in the Western Junggar region, NW China. Lithos 2012, 128, 73-83. [CrossRef]

49. Chen, J.F.; Han, B.F.; Ji, J.Q.; Zhang, L.; Xu, Z.; He, G.Q. Zircon U-Pb ages and tectonic implications of Paleozoic plutons in northern West Junggar, North Xinjiang, China. Lithos 2010, 115, 137-152. [CrossRef]

50. Zhang, F.; Zhang, Y.; Wang, D.K. Research Institute of Petroleum Exploration and Development, Company, S.O.; Geoscience, S.O. Identification of the lithology of Carboniferous and its reservoir characteristics in Chepaizi uplift, Junggar Basin. J. Southwest Pet. Univ. 2014, 36, 21-28.

51. Yang, F.; Mao, J.; Pirajno, F.; Yan, S.; Liu, G.; Zhou, G. A review of the geological characteristics and geodynamic setting of Late Paleozoic porphyry copper deposits in the Junggar region, Xinjiang uygur autonomous region, Northwest China. J. Asian Earth Sci. 2012, 49, 80-98. [CrossRef]

52. Didenko, A.N.; Morozov, O.L. Geology and paleomagnetism of middle-upper Paleozoic rocks of the Saur Ridge. Geotectonics 1999, 4, 64-80.

53. Vladimirov, A.G.; Kruk, N.N.; Khromykh, S.V.; Polyansky, O.P.; Chervov, V.V.; Vladimirov, V.G.; Travin, A.V.; Babin, G.A.; Kuibida, M.L.; Khomyakov, V.D. Permian magmatism and lithospheric deformation in the Altai caused by crustal and mantle thermal processes. Russ. Geol. Geophys. 2008, 49, 468-479. [CrossRef]

54. Zhu, Y.F.; Xu, X. The discovery of Early Ordovian ophiolite mélange in Taerbahatai Mts., Xinjiang, NW China. Acta Petrol. Sin. 2006, 22, 2833-2842.

55. Geng, H.Y.; Sun, M.; Yuan, C.; Zhao, G.C.; Xiao, W.J. Geochemical and geochronological study of Early Carboniferous volcanic rocks from the west Junggar: Petrogenesis and tectonic implications. J. Asian Earth Sci. 2011, 42, 854-866. [CrossRef]

56. Wang, R.; Zhu, Y.F. Geology of the Baobei Gold Deposit in Western Juggar and Zircon SHRIMP Age of Its Wall-Rocks, Western Junggar (Xinjiang, NW China). Geol. J. China Univ. 2007, 13, 590-602.

57. Guo, L.S.; Liu, Y.L.; Wang, Z.H.; Song, D.; Xu, F.J.; Su, L. The Zircon U-Pb LA-ICP-MS geochronology of volcanic rocks in Baogutu areas, Western Junggar. Acta Petrol. Sin. 2010, 26, 471-477.

58. An, F.; Zhu, Y.F. SHRIMP U-Pb zircon ages of tuff in Baogutu Formation and their geological significances. Acta Petrol. Sin. 2009, 25, 1437-1465.

59. Jian, P.; Liu, D.Y.; Shi, Y.R.; Zhang, F.Q. SHRIMP dating of SSZ ophiolites from northern Xinjiang Province, China: Implications for generation of oceanic crust in the Central Asian orogenic belt. In Structural and Tectonic Corre-lation across the Central Asia Orogenic Collage: North-Eastern Segment; Guide Book and Abstract Volume of the Siberian Workshop IGCP-480: Irkutsk-Ulan-Ude; Sklyarov, E.V., Ed.; Institute of the Earth's Crust of the Siberian Branch of Russian Academy of Sciences: Irkutsk, Russia, 2005; Volume 246.

60. Xu, Z.; Han, B.F.; Ren, R.; Zhou, Y.Z.; Zhang, L.; Chen, J.F. Ultramafic-mafic mélange, island arc and post-collisional intrusions in the Mayile Mountain, West Junggar, China: Implications for paleozoic intra-oceanic subduction-accretion process. Lithos 2012, 132-133, 141-161. [CrossRef]

61. Liu, X.; Xu, J.F.; Wang, S.Q.; Hou, Q.Y. Geochemistry and dating of E-MORB type mafic rocks from dalabute ophiolite in West Junggar, Xinjiang and geological implications. Acta Petrol. Sin. 2009, 25, 1373-1389.

62. Chen, B.; Zhu, Y.F.; An, F.; Qiu, T.; Chen, Y.C. The discovery of spinel peridotite in Baikouquan area, Karamay, Xinjiang. Geol. Bull. China. 2011, 30, 1017-1026.

63. Buckman, S.; Aitchison, J.C. Tectonic evolution of Palaeozoic terranes in West Junggar, Xinjiang, NW China. Geol. Soc. Lond. Special Pub. 2004, 226, 101-129. [CrossRef]

64. Feng, Q.W.; Li, J.Y.; Liu, J.F.; Zhang, J.; Qu, J.F. Ages of the Hongshan granite and intruding dioritic dyke swarms, in Western Junggar, Xinjiang, NW China: Evidence form LA-ICP-MS zircon chronology. Acta Petrol. Sin. 2012, 28, 2935-2949.

65. Jin, X.; Zhang, M.; Dong, G.S.; Zhu, X.G.; Xu, M.; Chen, Y. Preparation and structural study of metastable Mn phase grown on GaAs (OOI) substrate. MRS Proc. 1993, 326, 323. [CrossRef]

66. Zhao, P.; Xu, B.; Tong, Q.; Chen, Y.; Faure, M. Sedimentological and geochronological constraints on the Carboniferous evolution of Central Inner Mongolia, South Eastern Central Asian Orogenic Belt: Inland Sea deposition in a post-orogenic setting. Gondwana Res. 2016, 31, 253-270. [CrossRef] 
67. Su, Y.P.; Tang, H.F.; Hou, G.S.; Liu, C.Q. Geochemistry of aluminous a-type granites along darabut tectonic belt in West Junggar, Xinjiang. Geochimica 2006, 35, 55-67.

68. Kwon, S.T.; Tilton, G.R.; Goleman, R.C.; Feng, Y. Isotopic studies bearing on the tectonics of the West Junggar region, Xinjiang, China. Tectonics 1989, 8, 719-727. [CrossRef]

69. Gao, S.L.; He, Z.L.; Zhou, Z.Y. Geochenmical characteristics of the Karamay granitoids and their significance in West Junggar, Xinjiang. Xinjiang Geol. 2006, 24, 125-130.

70. Feng, Q.W.; Li, J.Y.; Liu, J.F.; Song, B.; Wang, Y.B.; Chen, W.; Zhang, Y. Ages and geological significance of the darkdykes emplaced in the Karamay pluton and adjacent area,in western Junggar, Xinjiang, NW China: Evidence form LA-ICP-MS zircon chronology and Ar-Ar amphibole chronology. Acta Petrol. Sin. 2012, 28, 2158-2170.

71. Kang, L.; Li, Y.J.; Zhang, B.; Zhang, H.W.; Wang, J.N. Petrographic evidence for magma mixing of Xiaerpu granite in West Junggar, Xinjiang. Acta Petrol. Mineral. 2009, 28, 423-432.

72. Liu, J.P.; Wang, H.; Li, S.H.; Tong, L.X.; Ren, G.L. Geological and geochemical features and geochronology of the Kayizi porphyry molybdenum deposit in the northern belt of Western Kunlun, NW China. Acta Petrol. Sin. 2010, 26, 3095-3105.

73. Ludwig, K.R. Eliminating mass-fractionation effects on U-Pb isochron ages without double spiking. Geochim. Cosmochim. Acta 2001, 65, 3139-3145. [CrossRef]

74. Zhang, Q.; Wang, Y.; Wang, Y.L. Preliminary study on the components of the lower crust in East China plateau during Yanshanian period: Constraints on $\mathrm{Sr}$ and $\mathrm{Nd}$ isotopic compositions of adakite-like rocks. Acta Petrol. Sin. 2001, 17, 505-513.

75. Fang, W.; Yang, S.; Liu, Z.; Wei, X.; Zhang, B. Geochemical characteristics and significance of major elements, trace elements and REE in mineralized altered rocks of large-scale tsagaan suvarga Cu-Mo porphyry deposit in Mongolia. J. Rare Earth 2007, 25, 759-769.

76. Hu, Z.C.; Liu, Y.S.; Gao, S.; Xiao, S.Q.; Zhao, L.S.; Günther, D.; Li, M.; Zhang, W.; Zong, K.Q. A “wire” signal smoothing device for laser ablation inductively coupled plasma mass spectrometry analysis. Spectrochim. Acta Part B 2012, 78, 50-57. [CrossRef]

77. Liu, W.; Liu, L.J.; Liu, X.J.; Shang, H.J.; Zhou, G. Age of the early devonian Kangbutiebao formation along the Southern Altay mountains and its Northeastern extension. Acta Petrol. Sin. 2010, 26, 387-400.

78. Defant, M.J.; Richerson, P.M.; De Boer, J.Z. Dacite genesis via both slab melting and differentiation: Petrogenesis of La Yeguada volcanic complex, Panama. J. Petrol. 1991, 32, 1101-1142. [CrossRef]

79. Defant, M.J.; Jackson, T.E.; Drummond, M.S.; De Boer, J.Z.; Bellen, H.; Feigeson, M.D.; Maury, R.C. The geochemistry of young volcanism throughout western Panama and southeastern Costa Rica: An overview. J. Geol. Soc. 1992, 149, 569-579. [CrossRef]

80. Defant, M.J.; Xu, J.F.; Kepezhinsksa, P.; Wang, Q.; Zhang, Q.; Xiao, L. Adakites: Some variations on a theme. Acta Petrol. Sin. 2002, 18, 129-142.

81. Middlemost, E.A.K. Naming materials in the magma igneous rock system. Earth-Sci. Rev. 1994, 37, $215-224$. [CrossRef]

82. Maniar, P.D.; Piccoli, P.M. Tectonic discrimination of granitoids. Geol. Soc. Am. Bull. 1989, 101, $635-643$. [CrossRef]

83. Sun, S.S.; Mcdonough, W.F. Chemical and Isotopic Systematics of Oceanic Basalts: Implications for Mantle Composition and Processes. Geol. Soc. Lond. Spec. Publ. 1989, 42, 313-345. [CrossRef]

84. Yin, J.Y.; Long, X.P.; Yuan, C.; Sun, M.; Zhao, G.C.; Geng, H.Y. A Late Carboniferous-Early Permian slab window in the West Junggar of NW China: Geochronological and geochemical evidence from mafic to intermediate dikes. Lithos 2013, 175-176, 146-162. [CrossRef]

85. Yin, J.Y.; Chen, W.; Yuan, C.; Yu, S.; Xiao, W.; Long, X. Petrogenesis of early Carboniferous adakitic dikes, Sawur region, northern West Junggar, NW China: Implications for geodynamic evolution. Gondwana Res. 2015, 27, 1630-1645. [CrossRef]

86. Yin, J.Y.; Chen, W.; Xiao, W.; Yuan, C.; Sun, M.; Tang, G. Petrogenesis of Early-Permian Sanukitoids from West Junggar, Northwest China: Implications for late Paleozoic crustal growth in central Asia. Tectonophysics 2015, 662, 385-397. [CrossRef]

87. Martin, H.; Smithies, R.H.; Rapp, R. An Overview of Adakite, Tonalite-Trondhjemite-Granodiorite (TTG), and Sanukitoid: Relationships and Some Implications for Crustal Evolution. Lithos 2005, 79, 1-24. [CrossRef] 
88. Castillo, P.R.; Janney, P.E.; Solidum, R.U. Petrology and geochemistry of Camiguin island, Southern Philippines: Insights to the source of Adakites and other lavas in a complex arc setting. Contrib. Mineral. Petrol. 1999, 134, 33-51. [CrossRef]

89. Atherton, M.P.; Petford, N. Generation of sodium-rich magmas from newly underplated basaltic crust. Nature 1993, 362, 144-146. [CrossRef]

90. Chung, S.L.; Liu, D.Y.; Ji, J.Q.; Chu, M.F.; Lee, H.Y.; Wen, D.J.; Lo, C.H.; Lee, T.Y.; Qian, Q.; Zhang, Q. Adakites from continental collision zones: Melting of thickened lower crust beneath southern Tibet. Geology 2003, 31, 1021-1024. [CrossRef]

91. Petford, N.; Atherton, M. Na-rich partial melts from newly underplated basaltic crust: The Cordillera Blanca Batholith, Peru. J. Petrol. 1996, 37, 1491-1521. [CrossRef]

92. Xu, H.J.; Ma, C.Q.; Ye, K. Early Cretaceous granitoids and their implications for the collapse of the Dabie orogen, eastern China: SHRIMP zircon U-Pb dating and geochemistry. Chem. Geol. 2007, 240, 238-259. [CrossRef]

93. Martin, H. Adakitic magmas: Modern analogues of Archaean granitoids. Lithos 1999, 46, 411-429. [CrossRef]

94. Rapp, R.P.; Shimizu, N.; Norman, M.D.; Applegate, G.S. Reaction between slab-derived melts and peridotite in the mantle wedge: Experimental constraints at 3.8 Gpa. Chem. Geol. 1999, 160, 335-356. [CrossRef]

95. Gao, S.; Rudnick, R.L.; Yuan, H.L.; Liu, X.M.; Liu, Y.S.; Xu, W.L.; Ling, W.L.; Ayers, J.; Wang, X.C.; Wang, Q.H. Recycling lower continental crust in the North China Craton. Nature 2004, 432, 892-897. [CrossRef]

96. Wang, Q.; Xu, J.F.; Jian, P.; Bao, Z.W.; Zhao, Z.Z.; Li, C.F.; Xiong, X.L.; Ma, J.L. Petrogenesis of adakitic porphyries in an extensional tectonic setting, Dexing, South China: Implications for the genesis of porphyry copper mineralization. J. Petrol. 2006, 47, 119-144. [CrossRef]

97. Streck, M.J.; Leeman, W.P.; Chesley, J. High-magnesian andesite from Mount Shasta: A product of magma mixing and contamination, not a primitive mantle melt. Geology 2007, 35, 351-354. [CrossRef]

98. Defant, M.J.; Drummond, M.S. Derivation of some modern arc magmas by melting of young subducted lithosphere. Nature 1990, 347, 662-665. [CrossRef]

99. Wang, J.L.; Zhang, Z.M.; Shi, C. Anatexis and dynamics of the southeastern Lhasa Terrane. Acta Petrol. Sin. 2008, 24, 1539-1551.

100. Macpherson, C.G.; Dreher, S.T.; Thirlwall, M.F. Adakites without slab melting: High pressure differentiation of island arc magma, Mindanao, the Philippines. Earth Planet. Sci. Lett. 2006, 243, 581-593. [CrossRef]

101. Whalen, J.B.; Currie, K.L.; Chappell, B.W. A-type granites: Geochemical characteristics, discrimination and petrogenesis. Contrib. Mineral. Pet. 1987, 95, 407-419. [CrossRef]

102. Eby, N.; Kochhar, N. Geochemistry and petrogenesis of the Malani igneous suite, north peninsular India. J. Geol. Soc. India 1990, 36, 109-130.

103. Hu, A.Q.; Jahn, B.M.; Zhang, G.X.; Chen, Y.B.; Zhang, Q.F. Crustal evolution and Phanerozoic crustal growth in Northern Xinjiang: Nd isotopic evidence. Part I. Isotopic characterization of basement rocks. Tectonophysics 2000, 328, 15-51. [CrossRef]

104. Tang, Y.J.; Zhang, H.F.; Deloule, E.; Su, B.X.; Ying, J.F.; Xiao, Y. Slab-derived lithium isotopic signatures in mantle xenoliths from northeastern North China Craton. Lithos 2012, 149, 79-90. [CrossRef]

105. White, D.J.; Musacchio, G.; Helmstaedt, H.H.; Harrap, R.M.; Thurston, P.C.; van der Velden, A.; Hall, K. Images of a lower-crustal oceanic slab: Direct evidence for tectonic accretion in the Archean Western Superior Province. Geology 2003, 31, 997-1000. [CrossRef]

106. Zagorevski, A.; Lissenberg, C.J.; Van Staal, C.R. Accretion of arc and backarc crust to continental margins: Inferences from the Annieopsquotch accretionary tract, Newfoundland Appalachians. Tectonophysics 2009, 479, 150-164. [CrossRef]

107. Tang, G.J.; Wyman, D.A.; Wang, Q.; Li, J.; Li, Z.X.; Zhao, Z.H. Asthenosphere-lithosphere interaction triggered by a slab window during ridge subduction: Trace element and Sr-Nd-Hf-Os isotopic evidence from Late Carboniferous tholeiites in the western Junggar area (NW China). Earth Planet. Sci. Lett. 2012, 329-330, 84-96. [CrossRef]

108. Gutscher, M.A. Geodynamics of flat subduction: Seismicity and tomographic constraints from the andean margin. Tectonics 2000, 19, 814-833. [CrossRef]

109. Kay, S.M.; Ramos, V.A.; Marquez, M. Evidence in Cerro Pampa Volcanic Rocks for slab-melting prior to Ridge-Trench collision in southern South America. J. Geol. 1993, 101, 703-714. [CrossRef] 
110. Yogodzinski, G.M.; Kay, R.W.; Volynets, O.N.; Koloskov, A.V.; Kay, S.M. Magnesian andesite in the Western Aleutian Komandorsky region: Implications for slab melting and processes in the mantle wedge. Geol. Soc. Am. Bull. 1995, 107, 505-519. [CrossRef]

111. Ma, X.H.; Chen, B.; Yang, M.C. Magma mixing origin for the Aolunhua porphyry related to Mo-Cu mineralization, eastern Central Asian Orogenic Belt. Gondwana Res. 2013, 24, 1152-1171. [CrossRef]

112. Pearce, J.A.; Harris, N.B.W.; Tindle, A.G. Trace element discrimination diagrams for the tectonic interpretation of granitic rocks. J. Petrol. 1984, 25, 956-983. [CrossRef]

113. Garfunkel, Z.; Anderson, C.A.; Schubert, G. Mantle circulation and the lateral migration of subducted slabs. J. Geophys. Res. 1986, 91, 7205-7223. [CrossRef]

114. Hamilton, W.B. Driving mechanism and 3-D circulation of plate tectonics. Geol. Soc. Am. Spec. Pap. 2007, 433, 1-25.

115. Elsasser, W.M. Sea-floor spreading as thermal convection. J. Geophys. Res. 1971, 76, 1101-1112. [CrossRef]

116. Uyeda, S.; Kanamori, H. Back-arc opening and the mode of subduction. J. Geophys. Res. Solid Earth 1979, 84, 1049-1062. [CrossRef]

117. Jiang, S.Y.; Zhao, K.D.; Jiang, Y.H.; Ling, H.F.; Ni, P. New type of tin mineralization related to granite in South China: Evidence from mineral chemistry, element and isotope geochemistry. Acta Geol. Sin. 2006, 22, 2509-2516.

118. Jiang, S.Y.; Ding, Q.; Yang, S.; Zhu, Z.; Sun, M.; Sun, Y. Discovery and significance of carbonate mud mounds from $\mathrm{Cu}$-Polymetallic deposits in the middle and lower Yangtze Metallogenic Belt: Examples from the Wushan and Dongguashan deposits. Acta Geol. Sin. 2011, 85, 744-756.

(C) 2020 by the authors. Licensee MDPI, Basel, Switzerland. This article is an open access article distributed under the terms and conditions of the Creative Commons Attribution (CC BY) license (http://creativecommons.org/licenses/by/4.0/). 